e-Journal of Educational

Research, Assessment and Evaluation

\section{REIIEVE}

Revista ELectrónica de Investigación y EValuación Educativa

\title{
EVALUACIÓN DEL IMPACTO DEL ESFUERZO FORMATIVO EN LA EMPLEABILIDAD DE LOS TRABAJADORES EN EL CONTEXTO DEL MODELO FORMATIVO TRIPARTITO ESPAÑOL
}

\section{[Evaluation of the impact of training effort in workers employability within the institutional Spanish training model]}

by/por

$\underline{\text { Article record }}$

$\underline{\text { About authors }}$

HTML format

\author{
Ramírez-del-Río, Antonio (antonio.ramirez@systeme.es) \\ Garrido Casas, Jorgina (jorgina.garrido@systeme.es)
}

$\underline{\text { Ficha del artículo }}$

$\underline{\text { Sobre los autores }}$

Formato HTML

\begin{abstract}
The association between quantity of training and employability was analysed through a non experimental research with two non equivalent groups. The operationalization of training quantity is considered to provide an indicator of training effort. Thus, training effort (number of courses) was analysed as the independent variable and employability as dependent variable (occupation indicators, activity enhancement and job performance). The results show that training is related to activity enhancement, specially when workers belong to a small/medium enterprise (SME) and/or to technical or qualified positions. A positive association between training and performance also appeared in the case of SMEs.
\end{abstract}

\section{Keywords}

Employability, labour market, social outcome, profesional training, training paradigm, training assessment, training results, training impact.

\section{Resumen}

Se analiza la asociación entre cantidad de formación y empleabilidad mediante una investigación no experimental con dos grupos no equivalentes. Se considera que la operativización de la cantidad de formación proporciona un indicador próximo al esfuerzo formativo realizado. Así, se estudió la variable independiente esfuerzo formativo (número de cursos) y la dependiente, empleabilidad (indicadores de ocupación, enriquecimiento de actividad y desempeño). Los resultados muestran que la formación está asociada al enriquecimiento de la actividad, sobre todo si los trabajadores pertenecen a una PYME y/o a puestos técnicos o cualificados. También hay una asociación positiva entre formación y desempeño en PYMES.

\section{Descriptores}

Empleabilidad, mercado de trabajo, retorno social, formación profesional, paradigma de formación, evaluación de la formación, resultados de la formación, impacto de la formación.

\section{Introducción}

En España, la crisis que atravesamos ha traído consigo un alto nivel de desempleo.
No es de extrañar que la formación y el impulso a la empleabilidad se hayan convertido en dos elementos determinantes de la ecuación pendiente de solucionar en lo que a polí- 
ticas socioeconómicas se refiere. En este contexto, el conocimiento de los resultados que producen los programas de formación adquiere especial importancia para los promotores de acciones formativas y para los encargados de planificar y gestionar la impartición de las mismas. Medir la repercusión de la formación facilita información para la adopción de decisiones técnicas y/o políticas sobre las acciones formativas a desarrollar, los colectivos a los que dirigirse, los métodos formativos o de gestión más adecuados, entre otras muchas cuestiones. En definitiva, la evaluación de la formación permite dotar de un mejor fundamento a las decisiones de gestión de la formación potenciando el logro de los mejores resultados. Por ejemplo, mejorar la capacitación de los trabajadores de forma continua, lograr su óptimo desarrollo profesional o mejorar y acelerar su incorporación al mercado laboral.

Se puede abordar la evaluación de los resultados de la formación desde dos enfoques: microscópico y macroscópico. Desde un enfoque microscópico la evaluación se centra en programas o acciones formativas concretas y en sus efectos en el individuo y en el trabajo que desarrollan, aunque en última instancia, también en el retorno económico para la empresa (ROI). Desde un enfoque macroscópico se valora los efectos de un conjunto de programas formativos de diferente naturaleza entre sí (plan de formación) con indicadores más globales de tipo socioeconómico referidos a empresas, sectores y grandes colectivos de trabajadores entre otros. Esta última perspectiva, más amplia en cuanto a número de acciones formativas incluidas en el análisis, es la que utilizamos. Asimismo, al hablar de los efectos, nos centramos en la repercusión en los trabajadores, es decir, una repercusión social más que económica, aunque ambas dimensiones no sean estrictamente separables una de la otra.

Recurriendo a una previa revisión bibliográfica, desde una orientación macroscópica se han realizado numerosos estudios en los que se ha valorado el efecto de la realización de acciones formativas en diferentes aspectos relacionados con el mundo laboral y las organizaciones: la inserción laboral (Puhani, 2002; Herrarte y Sáez, 2004; Nivorozhikin \& Nivorozhikin, 2005), la productividad (Dearden, Reed \& Van Reenen, 2000; Barrett \& O'Connell, 2001; Sutherland, 2004; Zwick, 2004; Hempell, 2005; Cassidy, Görg \& Strobl, 2005; Zwick, 2006), la mejora de la competitividad de la empresa (Van de Wiele, 2010; Molina \& Ortega, 2003), la variación de salarios (Bartel, 1995; Regner, 2002; Kuckulenz \& Zwick, 2003; Greenberg, Michalpoulos \& Robins, 2003; Budria \& Pereira, 2004; Arulampalam, Booth \& Bryan, 2004), la estabilidad laboral (Mamaqi y Miguel, 2009) y el rendimiento en el trabajo (Bartel, 1995; Boon Heng et al. 2006).

Los resultados de estas investigaciones, si bien tienden a ser favorables a la formación, no se manifiestan como contundentes en todos los casos. Como veremos, se trata de una conclusión que también es resultado del presente trabajo. Por otra parte, en todos ellos, como variable independiente se utiliza el realizar o no acciones formativas y cuando se habla de intensidad de la formación (Zwick, 2006), se alude al número de participantes en las mismas, un indicador que desde nuestro punto de vista se refiere al alcance de la formación. Como conclusión relevante para el presente artículo, en los estudios revisados no se hace referencia al número de acciones o número de horas como indicador de intensidad formativa. Mediante el presente estudio queremos profundizar un poco más en la relación formaciónempleabilidad, operativizando la realización de formación como variable independiente con indicadores de intensidad y/o cantidad de la misma.

A partir de aquí conviene concretar qué se entiende habitualmente por esfuerzo formativo. En primer lugar, sostenemos que se trata de un constructo multidimensional, del mismo modo que ocurre con el constructo de 
empleabilidad. Sin embargo, no es tan común en la literatura científica como lo es el constructo de empleabilidad. A pesar de todo, podemos afirmar que su uso en el mundo de la gestión profesional de la formación está bastante difundido. Una breve revisión en Internet en idioma español sirve para corroborar que su uso es frecuente, tanto en instituciones públicas como privadas, aludiendo sobre todo a una dimensión de carácter socioeconómico, soliendo aludir de una manera u otra a la inversión realizada en formación. Desde un ámbito algo más académico, un estudio de Pineda y Sarramona (2006) contiene un ejemplo del sentido socioeconómico dado al término. Por otra parte, estimamos que el término esfuerzo formativo también podría tener una acepción psicológica o motivacional. Ésta es la razón que nos lleva a considerar que estamos ante un constructo de naturaleza multidimensional, aunque como veremos en el presente trabajo solo utilizaremos un indicador (número de acciones formativas), siendo conscientes de la necesidad de mayor investigación en este sentido.

En cuanto al entorno en el que tiene lugar la formación analizada, es importante mencionar someramente el contexto institucional de los participantes y las acciones formativas objeto del estudio. La operativa de gestión formativa que ha centrado nuestra atención tiene lugar dentro del modelo español de formación continua cofinanciada, cuya gestión al más alto nivel es de carácter tripartito (Administración, Patronal y Sindicatos). No es ni mucho menos el objetivo del presente trabajo explicar en qué consiste dicho sistema de gestión, pero sí conviene especificar que el plan de formación que mayoritariamente alimenta los datos con los que se ha trabajado se enmarca en la modalidad de Formación de Oferta (versus Formación de Demanda). De una manera más concreta, se trata de un Plan Formativo Autonómico Intersectorial, siendo su promotor un agente social sobre el cual no se especifica si es un sindicato o una asociación empresarial por motivos de confidencialidad.
En términos generales, los autores del presente trabajo consideramos que la operativa del sistema nacional de formación puede caracterizarse como doble y frecuentemente contradictorio: un paradigma orientado al alcance y otro orientado al impacto. Por un lado, aquel cuya lógica consiste en lograr el mayor alcance posible de la formación entre trabajadores y empresas, dando por sentado su efecto beneficioso; por otro, aquel centrado en obtener el mejor resultado posible en términos socioeconómicos. El concepto de paradigma se utiliza en el sentido formulado por Kuhn (1962). Así, se destaca la convivencia de dos modelos operativos. Estas conclusiones se contrastaron en un trabajo de diagnóstico cualitativo solicitado por la FEMP (2009) con la colaboración de Griker Orgemer - Ramírez del Río en el ámbito de las Administraciones Locales. Sin ánimo de cuantificar su influencia o extensión, y a pesar de que constituyen una línea de investigación diferente al eje central del presente trabajo, se mencionan con el único fin de contextualizar la gestión y/o la praxis de la formación analizada, además de servir como consideración interpretativa posterior.

Pero existen otras variables que también configuran el entorno en el cual tiene lugar la formación y que igualmente modulan los resultados de la misma. Por un lado, nos referimos a las variables que provienen del contexto socioeconómico y son ajenas a la formación (sector productivo, tamaño de empresas, colectivos de trabajadores...). Por otro, las variables propias del tratamiento formativo (modalidades presencial, a distancia, on-line, naturaleza transversal o específica de los contenidos, duración de los programas, carga práctica...). Pues bien, dado el foco socioeconómico del estudio, el presente trabajo se ha centrado exclusivamente en las variables ajenas a la formación. De este modo, se opta por dejar para un momento posterior lo que podría ser una investigación más específica que relacionara el efecto de la formación en función de las características de la misma formación. Quedaría para el 
futuro la conjunción de ambas influencias en lo que podría ser un avance hacia un modelo teórico de la influencia de la formación en la empleabilidad.

Respecto al constructo de empleabilidad, la literatura revisada coincide en que éste ha variado a lo largo de la historia y resulta difícil definirlo ya que obedece a la interrelación de múltiples variables y disciplinas. Entre las múltiples definiciones que se han dado del mismo destacamos su esencia en lo siguiente:

- Hillage y Pollard (1998): Una dimensión de la vida laboral de las personas que refiere la capacidad para obtener un trabajo, mantenerse en él y progresar en una organización contribuyendo exitosamente a ésta.

- Finn (2000): Habilidad para "ser empleado". Se puntualizan tres elementos: obtener un empleo inicial, la habilidad para mantenerlo y la habilidad para movilizarse hacia otros empleos.

- Knight y Yorke (2003): Conjunto de logros y atributos personales, que hacen que los individuos tengan la probabilidad de obtener un empleo y tengan éxito en los espacios donde se desempeñan.

Siguiendo las definiciones señaladas, en el presente estudio definimos el constructo de empleabilidad como la capacidad/habilidad para la consecución y mantenimiento del empleo, así como el logro de mejoras en el mismo a lo largo de la vida laboral de las personas. Partiendo de esta definición, concretamos la medición de la empleabilidad en tres dimensiones cuyas posibilidades de visión conjunta y comparativa consideramos interesantes: socio-económica (consecución o mantenimiento de la ocupación), organizacional (mejora de la actividad o rol) e interindividual (mejora en el desempeño). Esta estructura es consecuente con la tríada sociedad-empresa-persona puesta de manifiesto en el documento resultante del Encuentro sobre Empleabilidad (1999) de FUNDIPE que fue redactado por los Profesores Sáez y Torres de la Universidad Autónoma de Madrid.

La estructura seguida presenta las siguientes características en relación con la empleabilidad:

- Ocurrir en la persona, siendo algo observable en el propio individuo. En efecto, la definición se centra en la vida laboral de las personas. Si bien se admite la posibilidad de que existan condicionantes en la vida laboral que no provengan exclusivamente del individuo.

- Ser sensible a la situación laboral del trabajador, abarcando tanto a empleados como a desempleados. Algo que está implícito en la definición al referirnos a la consecución y mantenimiento del empleo.

- Ser completo y multifactorial. En la práctica, el modelo abarca diversas dimensiones o factores, desde el individuo hasta su entorno. Concretamente, se establecen tres ámbitos de evaluación cuya simultaneidad puede arrojar una interesante visión de conjunto:

- La consecución y el mantenimiento del empleo

- La mejora de la actividad o rol profesional

- La mejora en el nivel de desempeño

A lo que cabría añadir otro tipo de mejoras particulares. Sin ir más lejos, la inclusión de condiciones laborales como el salario. Un indicador que sí aparece en la revisión bibliográfica realizada, pero que no se ha incluido como parte del estudio, aunque podría considerarse en un trabajo posterior.

- Permitir una fácil conversión operativa de los conceptos recurriendo a indicadores cuantitativos directamente observables $u$ objetivos. Los tres ámbitos por los que se puede medir la empleabilidad de una persona admiten la ideación de indicadores 
duros formulados en términos cuantitativos.

Con el fin de contextualizar la hipótesis del presente trabajo en el entorno social vigente, es conveniente tener en cuenta que se destinan ingentes cantidades de recursos públicos a la formación. Por tanto, existe un indiscutible interés social en la realización de trabajos sistemáticos de evaluación de la formación que permitan conocer el retorno de estas políticas. En este contexto, siendo coherentes con el planteamiento teórico realizado hasta el momento, hipotetizamos que existe una asociación positiva y significativa entre el esfuerzo formativo (variable causal) y la empleabilidad de los trabajadores (variable efecto).

De este modo:

a) Como primer resultado, cabe esperar alguna evidencia significativa de impacto de la formación en la consecución o mantenimiento de la ocupación en los trabajadores.

b) Como segundo resultado, y en una proporción mayor que el resultado anterior, se estima que hay una asociación positiva y significativa entre formación y enriquecimiento de la actividad o rol que desempeña el trabajador.

c) Como tercer resultado, esperamos la mayor asociación positiva y significativa entre formación y desempeño laboral.

Algunas consideraciones teóricas previas que podrían enmarcar las hipótesis anteriores son:

a) La consecución y el mantenimiento del empleo es un propósito principal que persigue la administración pública y los agentes sociales. Asumiendo que el nivel de empleo es la variable más sensible a la situación económica, cabe esperar que la asociación con la formación no sea grande; pero cuando menos, sí significativa. b) En cuanto al enriquecimiento de la actividad, el individuo debería tener un papel más relevante, aunque también cabe que la dinámica de la empresa y la situación económica influyan de una manera decisiva. Asimismo, es posible que tanto en una situación económica favorable como desfavorable, en las organizaciones se sigan produciendo movimientos de promoción o asunción de nuevas funciones/tareas.

c) Por último, en lo que se refiere al desempeño laboral, cabe pensar que el propio individuo tiene el papel más relevante en la variación de este indicador. $\mathrm{Su}$ capacidad para influir en esta variable es mayor en tanto que el desempeño es una consecuencia más directa de la persona, y no tan dependiente del entorno económico.

\section{Método de investigación}

Para analizar la asociación entre el esfuerzo formativo y la empleabilidad planteada en la hipótesis se recurre a la investigación correlacional ex post facto. En concreto, utilizamos un diseño no-experimental con dos grupos no equivalentes sin pretest. La asignación de los sujetos a cada uno de los grupos en este tipo de diseños no es aleatoria, lo que supone una amenaza para la validez interna. Esta dificultad se compensó prestando especial atención a aquellas variables que, ajenas a la formación, podían incidir en los efectos de la acción formativa tales como: tamaño de la empresa, sector económico, área funcional en la que el participante desarrolla su actividad laboral, etc. Todas ellas variables que concretamos en el apartado siguiente.

\section{Variables e indicadores utilizados}

Para definir el esfuerzo formativo de manera operativa se recurrió inicialmente a dos indicadores: el número de acciones formativas, y el número de horas de formación realizadas. Para cada uno de ellos se establecieron dos niveles: bajo y alto a partir de los 
cuartiles obtenidos. Los niveles de esfuerzo quedaron establecidos de la siguiente manera, si bien, como veremos luego en el apartado de Resultados Obtenidos, finalmente se trabajó con un único indicador, el de "número de acciones":

- Bajo esfuerzo $\left(\mathrm{Q}_{1}\right)$ :

- Número de acciones: 1 acción formativa realizada

- Número de horas: 41 horas o menos

- Alto esfuerzo $\left(\mathrm{Q}_{3}\right)$ :

- Número de acciones: 2 o más acciones formativas realizadas

- Número de horas: 200 horas o más
Partiendo de la definición de empleabilidad expresada (consecución y mantenimiento del empleo, logro de mejoras en el mismo a lo largo de la vida laboral y desempeño) se utilizaron las siguientes variables de empleabilidad:

- Consecución de empleo

- Mantenimiento de la ocupación

- Enriquecimiento del trabajo

- Desempeño laboral

Finalmente, las variables e indicadores utilizados en el estudio para cada una de ellas se presentan en la Tabla 1.

Tabla 1. Variables e indicadores del estudio

\begin{tabular}{|c|c|c|}
\hline Tipo de variable & Denominación & Indicador \\
\hline Independiente & Esfuerzo formativo & $-\mathrm{N}^{\mathrm{o}}$ de acciones formativas realizadas \\
\hline \multirow{6}{*}{ Moduladoras } & \multirow{2}{*}{ Tipo de empresa } & -Tamaño \\
\hline & & -Sector económico \\
\hline & \multirow{2}{*}{$\begin{array}{l}\text { Tiempo en la situación laboral } \\
\text { actual }\end{array}$} & -Tiempo ocupado \\
\hline & & -Tiempo desempleado \\
\hline & \multirow{2}{*}{ Actividad profesional } & -Área funcional \\
\hline & & -Pesto de trabajo \\
\hline \multirow{5}{*}{$\begin{array}{l}\text { Dependiente } \\
\text { (Empleabilidad } \\
\text { como variable } \\
\text { multidimensional) }\end{array}$} & \multirow{2}{*}{$\begin{array}{l}\text { Consecución de empleo }(\mathrm{CO}) / \\
\text { Mantenimiento de ocupación } \\
(\mathrm{MO})\end{array}$} & -\% desocupados que pasan a situación de ocupados \\
\hline & & -\% ocupados que se mantienen ocupados \\
\hline & \multirow{2}{*}{ Enriquecimiento del trabajo } & -\% que asciende de categoría profesional \\
\hline & & -\%que amplía sus funciones y/o responsabilidades \\
\hline & Desempeño laboral & -\% que mejora el desempeño en puesto de trabajo \\
\hline
\end{tabular}

Todos los indicadores tienen una base objetiva salvo el referente al desempeño laboral, donde existe cierto componente de subjetividad. Aun con todo, en este caso, únicamente se registró la observación cuando los encuestados, previamente, respondieron positivamente a la pregunta de si existe en su empresa un sistema formalmente establecido de evaluación del desempeño.

\section{Control de variables moduladoras}

No pudiéndose aplicar los mecanismos típicos para controlar el efecto de las variables moduladoras (aleatorización y/o mantenimiento constante) propio de los diseños experimentales, se decidió establecer la asociación existente de estas variables con la relación esfuerzo formativo-empleabilidad. De este modo se obtuvo una información adicional útil para contextualizar la interpretación de los resultados. Como ya se ha indicado en la Introducción, esta información se limitó a las consideradas variables moduladoras ajenas a la formación y que se presentan en la Figura 1. 


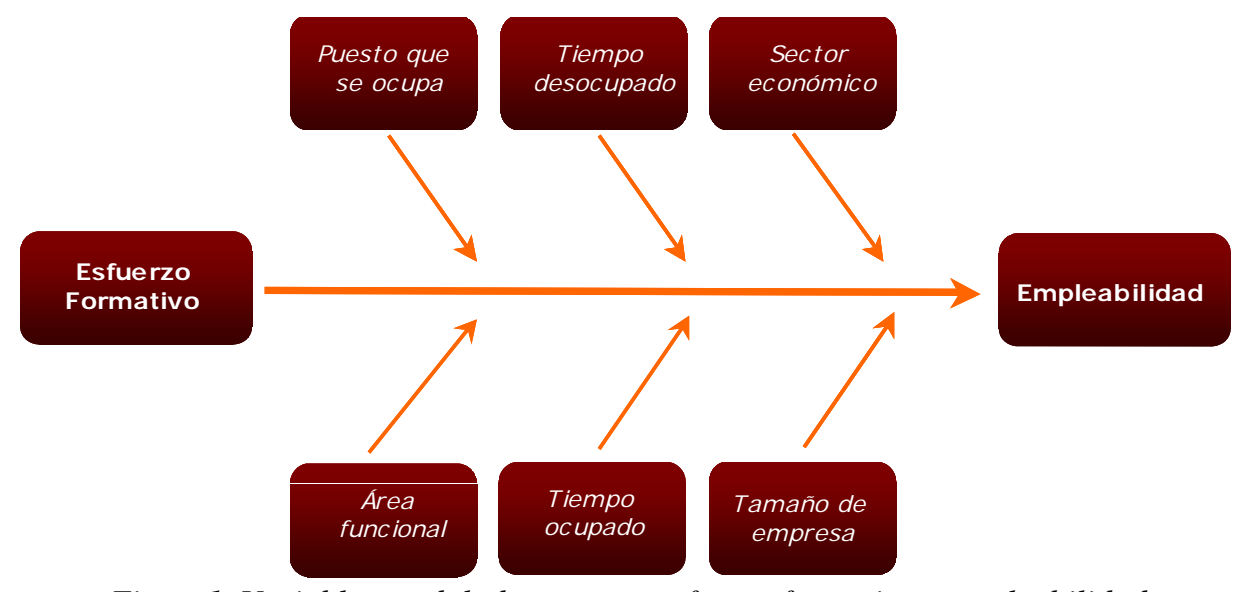

Figura1. Variables moduladoras entre esfuerzo formativo y empleabilidad

\section{Diseño de investigación}

Como hemos mencionado anteriormente, se utilizó un diseño en el que se cruzaron el esfuerzo formativo y la empleabilidad, utili- zando los indicadores ya mencionados. El diseño del estudio se plasma tal como aparece en la Tabla 2.

\begin{tabular}{|c|c|c|c|c|}
\hline \multirow{2}{*}{$\begin{array}{r}\text { Variables de } \\
\text { Empleabilidad }\end{array}$} & \multirow{2}{*}{$\begin{array}{l}\text { Tabla 2. Diseño de invest } \\
\text { MCO=Mantenimiento Consecución } \\
\text { Ocupación } \\
\text { EA=Enriquecimiento de la Actividad } \\
\text { DL=Desempeño Laboral }\end{array}$} & \multicolumn{3}{|c|}{ EMPLEABILIDAD } \\
\hline & & MCO & EA & DL \\
\hline \multirow{2}{*}{$\begin{array}{l}\text { FORMACIÓN } \\
\text { (Esfuerzo formativo) }\end{array}$} & Alto Esfuerzo Formativo $(\mathrm{Q} 3 \uparrow)$ & & & \\
\hline & Bajo Esfuerzo Formativo $(Q 1 \downarrow)$ & & & \\
\hline
\end{tabular}

\section{Fuentes de información y trabajo de campo}

Se obtuvo la información a partir de la realización de un cuestionario telefónico de respuestas cerradas, aplicado a los participantes en acciones formativas promovidas por una organización promotora de Convenios de Formación Intersectorial de carácter autonómico durante el año 2009. El estudio se realizó con los datos proporcionados por Esentia, Innovación, Seguridad y Desarrollo S.L. La utilización de un cuestionario telefónico permitió reducir los costes del estudio, y a la vez, facilitar un acceso más rápido y eficaz a los participantes. Adicionalmente, se incluyeron algunas preguntas sobre el conocimiento de la entidad promotora y los motivos de abandono de la formación relevantes para la entidad pero que no forman parte del estudio.
El cuestionario estaba estructurado en tres grandes apartados: a) datos identificativos del participante en la acción formativa (edad, sexo, categoría profesional), b) datos identificativos de la formación realizada (denominación de la acción formativa, modalidad, área temática, número de horas, fecha de finalización) y c) datos sobre empleabilidad (ascenso, cambio de funciones, situación laboral...) y otras variables moduladoras de la formación. La información de los dos primeros apartados se completó a partir de las bases de datos facilitadas por la propia entidad promotora. Ello supuso un ahorro de tiempo en las entrevistas a la vez que proporcionó mayor fiabilidad a los datos. Únicamente en el caso de que los participantes hubieran realizado formación adicional con otras entidades, se caracterizó dicha formación mediante el cuestionario. Los contenidos enmarcados en la estructura anterior res- 
ponden a las variables fijadas a priori para medir el impacto de la formación.

Con la finalidad de evitar subjetividades derivadas de opiniones por parte de los encuestados respecto a los efectos de la formación que recibieron, se recogió información basada en hechos concretos ocurridos en la trayectoria profesional del trabajador desde la realización de la última formación recibida. De esta forma sobre la movilidad laboral, por ejemplo, se incluyeron cuestiones tales como: ¿Desde que finalizó la última acción formativa ha cambiado de categoría profesional? ¿Desde que finalizó la última acción formativa han variado sus funciones o responsabilidades? ¿Ha mantenido sus funciones o responsabilidades? ¿Se han ampliado? ¿Han cambiado? Gracias a este tipo de preguntas logramos que los resultados obtenidos respondan a cambios reales de la situación de los participantes, y no tanto a sus percepciones (Ver cuestionario en Anexo).

\section{Trabajo de campo}

El trabajo de campo se llevó a cabo en los meses de marzo y abril de 2009, finalizando durante la primera semana de mayo. Se estableció un sistema de llamadas personalizado, adecuado a las características del informante, de manera que, en muchos casos, permitió concertar citas telefónicas para cumplimentar el cuestionario. Se realizó una media aproximada de 3 llamadas por informante lo que supuso un total de 1.526 llamadas, de las cuales, el $54,6 \%$ resultaron fallidas, bien por números de teléfono erróneos, rechazo del interlocutor a participar en el estudio o "Sin contacto" tras varios intentos.

\section{Muestra de participantes y acciones formativas}

Se utilizó para realizar el estudio, el listado de participantes en acciones formativas realizadas por una entidad promotora de la Comunidad de Madrid, durante el año 2009. Esto es, un total de 932 participantes en acciones formativas. Se pudo contactar y accedieron a responder al cuestionario diseñado un $47,75 \%$ de ellos, con lo que la muestra quedó constituida, finalmente, por 445 participantes. El perfil demográfico de los participantes se muestra en la Tabla 3.

Tabla 3. Perfil demográfico de la muestra de participantes

\begin{tabular}{lccc}
\hline Grupos de edad & $\%$ & Sexo & $\%$ \\
\hline 29 años o menos & 25,0 & Hombres & 55,9 \\
30-39 años & 43,7 & Mujeres & 44,1 \\
40-49 años & 19,7 & & \\
50 años o más & 9,0 & & \\
Nc & 2,6 & & \\
\hline
\end{tabular}

En la Tabla 4 se clasifica la muestra según dos indicadores de esfuerzo formativo. Realizaron dos o más acciones formativas (alto esfuerzo formativo) un total de 275 participantes y doscientas o más horas de formación (alto esfuerzo formativo), 128 participantes. Esta primera diferencia de casos se debe a que cuando se usó el indicador "número de horas" se eliminaron los valores intermedios $\left(\mathrm{Q}_{2}\right.$ y $\left.\mathrm{Q}_{3}\right)$, operación que no tuvo lugar al utilizar el indicador "número de acciones formativas". Pero como veremos seguidamente, hay más razones que justifican las diferencias entre las muestras de los análisis específicos.

Tabla 4. Caracterización de la muestra de participantes según esfuerzo formativo

\begin{tabular}{lclc}
\hline \multicolumn{4}{c}{ Esfuerzo formativo } \\
\hline $\begin{array}{l}\text { Acciones } \\
\text { formativas }\end{array}$ & $\begin{array}{l}\text { Partici- } \\
\text { pantes }\end{array}$ & $\begin{array}{l}\text { Horas de } \\
\text { formación }\end{array}$ & $\begin{array}{l}\text { Partici- } \\
\text { pantes }\end{array}$ \\
\hline $\begin{array}{l}\text { Alto } \\
\text { esfuerzo }\end{array}$ & 275 & $\begin{array}{l}\text { Alto } \\
\text { esfuerzo }\end{array}$ & 128 \\
$\begin{array}{l}\text { Bajo } \\
\text { esfuerzo }\end{array}$ & 172 & $\begin{array}{l}\text { Bajo } \\
\text { esfuerzo }\end{array}$ & 95 \\
\hline
\end{tabular}

Por otro lado, no siempre coincide la muestra de análisis con la muestra total del estudio (en la práctica, 445 casos al usarse el indicador "número de acciones formativas"). Esto es debido a varias causas:

- Los encuestados no siempre responden a todas las preguntas del cuestionario, por ello varía la base de análisis, bien debido a los filtros existentes en el cuestionario, o bien a la negativa del encuestado. Por 
ejemplo, en la Figura 2 ("Relación entre el esfuerzo formativo y variación de la situación laboral de los ocupados") la muestra de análisis es de 335 participantes. No se incluyen en este análisis el grupo de participantes desocupados $(\mathrm{n}=103)$. La suma de ambos grupos es de 438 próxima a 445 de la muestra inicial. Siete de los encuestados no respondieron a esta pregunta.

- Se utilizan en el análisis únicamente los intervalos extremos de la variable. Por ejemplo en la Figura 4 ("Relación entre tiempo ocupado y esfuerzo formativo. Ocupado-ocupado") además de analizar lo que ocurre únicamente en el grupo de par- ticipantes que se mantienen ocupados $(\mathrm{n}=291)$ se estudia la asociación del esfuerzo formativo con los dos valores extremos del tiempo ocupado, 36 meses o menos y 61 meses o más. Una vez más se eliminan del análisis los valores intermedios, es decir, los sujetos cuyo tiempo ocupado es de 37 a 60 meses.

Debido a estas variaciones muestrales respecto al grupo inicial de participantes entrevistados, se indica en el pie de las Figuras, incluidas en el apartado de Resultados, la base de participantes (n) a partir de los cuales se calcularon los resultados en cada caso.

Tabla 5. Caracterización de la muestra de acciones formativas

\begin{tabular}{|c|c|c|c|}
\hline \multicolumn{4}{|c|}{ Áreas temáticas del conjunto de las acciones formativas } \\
\hline & $\%$ & Tipo* & $\%$ \\
\hline Administración/Gestión Inmobiliaria & 3,8 & $\mathrm{E}$ & \\
\hline Comunicación audiovisual & 6,5 & $\mathrm{E}$ & \\
\hline Contabilidad y finanzas & 11,7 & $\mathrm{E}$ & \\
\hline Diseño gráfico & 3,6 & $\mathrm{E}$ & \\
\hline Estética & 6,0 & $\mathrm{E}$ & \\
\hline Explosivos & 1,8 & $\mathrm{E}$ & 54,3 \\
\hline Marketing/Gestión de eventos & 3,3 & $\mathrm{E}$ & \\
\hline Mediación de seguros & 6,1 & $\mathrm{E}$ & \\
\hline Vigilancia y Seguridad & 8,3 & $\mathrm{E}$ & \\
\hline Servicios Sociales & 3,2 & $\mathrm{E}$ & \\
\hline Gestión y dirección de empresas & 1,1 & $\mathrm{~T}$ & \\
\hline Habilidades directivas & 3,5 & $\mathrm{~T}$ & \\
\hline Idiomas & 15,4 & $\mathrm{~T}$ & \\
\hline Informática & 9,6 & $\mathrm{~T}$ & 35,4 \\
\hline Protección de Riesgos Laborales & 3,9 & $\mathrm{~T}$ & \\
\hline Técnicas de venta/negociación & 1,9 & $\mathrm{~T}$ & \\
\hline Otros & 10,3 & $\mathrm{~T}$ & 10,3 \\
\hline Total & 100 & & 100 \\
\hline
\end{tabular}

*E: Acciones Específicas; T: Acciones Transversales

Es de interés conocer a grandes rasgos en qué consistieron las acciones formativas, las cuales se muestran en la Tabla 5. Como se puede observar en la misma, las acciones formativas realizadas con mayor frecuencia fueron las de Idiomas $(15,4 \%)$ y Contabilidad y Finanzas $(11,7 \%)$. En conjunto, predominaron las acciones formativas específicas $(54,3 \%)$.

\section{Análisis estadístico}

En éste apartado se indican las principales técnicas inferenciales utilizadas. El nivel de confianza en todos los análisis para determinar la significación estadística fue del $95 \%$. El programa de procesamiento estadístico utilizado fue el SPSS 13.0. En un primer momento, para determinar qué indicador de esfuerzo formativo utilizar en el estudio se realizó un análisis de regresión logística múltiple. Como variables dependientes del "número de acciones formativas" y "número de horas de formación" se incluyeron los indicadores elegidos para operativizar el constructo de empleabilidad en sus diferentes dimensiones (consecución y mantenimiento 
de la ocupación, mejora del trabajo o rol, y variación en el nivel de desempeño). Se siguió en este análisis el método paso por paso.

Partiendo del mencionado análisis previo se llevaron a cabo las diversas asociaciones entre el esfuerzo formativo y las diversas variables de empleabilidad. Dado que la variable dependiente y las independientes se basan en escalas nominales, se utilizó el contraste Chi-cuadrado para determinar si las diferencias en empleabilidad en los diferentes grupos de esfuerzo formativo eran atribuibles al azar o por el contrario había una asociación entre las mismas. Como valor del estadístico se utilizó en todos los análisis la Corrección de Continuidad de Yates (Colton, 1979; Domènech, 1995) como método más restrictivo que el Coeficiente de Correlación de Pearson.

Asimismo, se hizo un análisis adicional para analizar el efecto de las variables moduladoras (únicamente en el caso de que los resultados anteriores fueran estadísticamente significativos) se realizó un análisis utilizando el estadístico Chi-cuadrado, nuevamente debido a la naturaleza nominal de las escalas. Se estudiaron las variables objeto del estudio controladas por los diferentes valores de una tercera variable (tamaño de empresa, catego- ría laboral, sector económico...). En los casos en que se observó una asociación estadísticamente significativa al incluir la variable moduladora, se calculó la intensidad de la asociación utilizando la razón de proporciones (Risk estimate) (Domènech, 1995).

\section{Resultados}

En este apartado nos limitamos a describir los resultados tal como se obtuvieron de la explotación estadística, dejando para el apartado de Conclusiones su interpretación.

\section{Selección de indicador de esfuerzo formativo}

Como ya se ha señalado anteriormente, para determinar en qué medida los indicadores de esfuerzo formativo eran sensibles a la variación de la empleabilidad se realizó un análisis de regresión logística múltiple en el que se utilizaron como variables independientes el número de acciones formativas $\mathrm{y}$ el número de horas de formación. Como consecuencia, únicamente la variable "número de acciones formativas" resultó ser un predictor fiable de algunas de las variables que se incluyen en el constructo de empleabilidad. Éstas fueron "promoción o ascenso" y "cambio de funciones/responsabilidades" (Tablas 6 y 7 ).

Tabla 6. Modelo de regresión de la influencia del esfuerzo formativo en la promoción (Ascenso, No ascenso). Paso 1

\begin{tabular}{lllllll}
\hline & $\mathbf{B}$ & $\begin{array}{c}\text { Error } \\
\text { estándar }\end{array}$ & $\begin{array}{c}\text { Prueba } \\
\text { de Wald }\end{array}$ & $\begin{array}{c}\text { Grados de } \\
\text { libertad }\end{array}$ & Sig. & Exp(B) \\
\hline $\mathbf{N}^{\mathbf{0}}$ de acciones formativas completas & 1,384 &, 649 & 4,549 & 1 &, 033 & 3,991 \\
$\mathbf{N}^{\mathbf{0}}$ de horas de formación realizadas &, 759 &, 713 & 1,131 & 1 &, 288 & 2,135 \\
Constante & 1,382 &, 367 & 14,177 & 1 &, 000 & 3,982 \\
\hline N=153; Porcentaje pronosticado: $90,8 \%$ & \multicolumn{5}{l}{} \\
Sensibilidad: 0\% Especificidad: $100 \%$ Chi-square: $8,971, \mathrm{p}=0,011, \mathrm{R}^{2}$ de Nagelbekerke: 0,124 & \\
\hline
\end{tabular}

Tabla 7. Modelo de regresión de la influencia del esfuerzo formativo en el cambio de funciones y/o Responsabilidades (Sí cambio, No cambio). Paso 1

\begin{tabular}{lllllll}
\hline & B & $\begin{array}{l}\text { Error } \\
\text { estándar }\end{array}$ & $\begin{array}{l}\text { Prueba } \\
\text { de Wald }\end{array}$ & $\begin{array}{l}\text { Grados de } \\
\text { libertad }\end{array}$ & Sig. & Exp(B) \\
\hline $\mathbf{N}^{\mathbf{0}}$ de acciones formativas completas & 1,207 &, 492 & 6,009 & 1 &, 014 & 3,343 \\
$\mathbf{N}^{\mathbf{0}}$ de horas de formación realizadas &, 197 &, 508 &, 151 & 1 &, 698 & 1,218 \\
Constante &, 961 &, 321 & 8,959 & 1 &, 003 & 2,615 \\
\hline $\begin{array}{l}\mathrm{N}=153 \text {; Porcentaje pronosticado: } 84,3 \% \\
\text { Sensibilidad: } 0 \% \text { Especificidad: } 100 \% \text { Chi-square: } 8,070, \mathrm{p}=0,018, \mathrm{R}^{2} \text { de Nagelbekerke: } 0,088\end{array}$ \\
\hline
\end{tabular}




\section{Resultados del esfuerzo formativo en la empleabilidad de los trabajadores}

Siguiendo las dimensiones incluidas en la definición operativa de la empleabilidad, esto es, impacto en los aspectos socioeconómicos, organizacionales y personales, presentamos los resultados obtenidos agrupados en tres apartados.

\section{Dimensión socioeconómica: Consecución} o mantenimiento de la ocupación

La variación o mantenimiento de la situación laboral no muestra una asociación significativa con el esfuerzo formativo realizado. La consecución o mantenimiento de la ocupación es independiente del esfuerzo formativo. Esta relación no varía con la inclusión de las variables moduladoras en el análisis.
Variación de la situación laboral de los ocupados

Entre los ocupados, más del $80 \%$ mantienen su puesto de trabajo desde la última acción formativa realizada, independientemente del esfuerzo formativo realizado (Figura 2). No obstante, cabe mencionar que, a pesar de la falta de significación estadística, aquellos que han realizado mayor esfuerzo formativo, varían con más frecuencia su situación laboral de ocupado a desocupado. El 17,07\% de los ocupados que pasan a una situación de desocupado han realizado un alto esfuerzo formativo, 6,59 puntos porcentuales por encima de los que han realizado un esfuerzo formativo bajo.

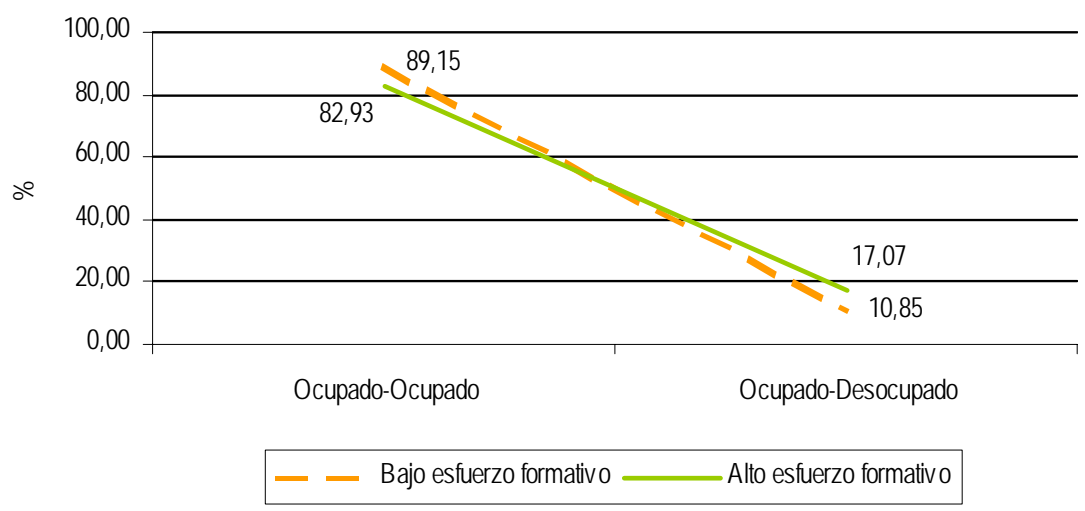

Base: Ocupado-Ocupa: $n=291$; Ocupado-desocupad: $n=44$

Figura 2. Relación entre esfuerzo formativo y variación de la situación laboral de los ocupados

Debido a la relevancia de ésta tendencia negativa entre esfuerzo formativo y variación de la situación laboral de los ocupados, profundizamos en el análisis estudiando de forma independiente el grupo de trabajadores ocupados que se mantienen ocupados (Ocupado-Ocupado) y el de ocupados que pasan a una situación de desocupados (OcupadoDesocupado). Analizamos en cada uno de los grupos, de manera independiente, la asociación entre esfuerzo formativo y las diferentes variables consideradas moduladoras, esto es: tamaño de empresa, sector económico, área funcional en la que se desarrolla la actividad laboral en la empresa, tipo de puesto de trabajo, tiempo ocupado y tiempo desocupado.

\section{Variables asociadas al esfuerzo formativo} en trabajadores ocupados

En la categoría de ocupados que se mantienen ocupados, las variables que se encuentran asociadas al esfuerzo formativo son las de "área funcional" y "tiempo ocupado". Los trabajadores que se encuentran en el área funcional de producción con mayor frecuencia realizan un alto esfuerzo formativo que aquellos que desarrollan su actividad laboral en otras áreas funcionales (Chicuadrado=7,893; $\mathrm{p}=0,005)$. El 50,79\% de los 
trabajadores del área de producción realizan un alto esfuerzo formativo, frente a un $30,70 \%$ en el caso de aquellos que se encuentran en otras áreas funcionales (Figura $3)$.

Respecto al tiempo ocupado en el puesto de trabajo, se observa que aquellos que llevan en su puesto menos tiempo (36 meses o menos) con mayor frecuencia realizan un alto esfuerzo formativo (Chicuadrado=5,026; $p=0,025)$. El 42,57\% de los que llevan en su puesto de trabajo 3 años o menos realizan un alto esfuerzo formativo, lo que ocurre, únicamente en un $27,42 \%$ en el caso de aquellos que llevan cinco años o más (Figura 4).

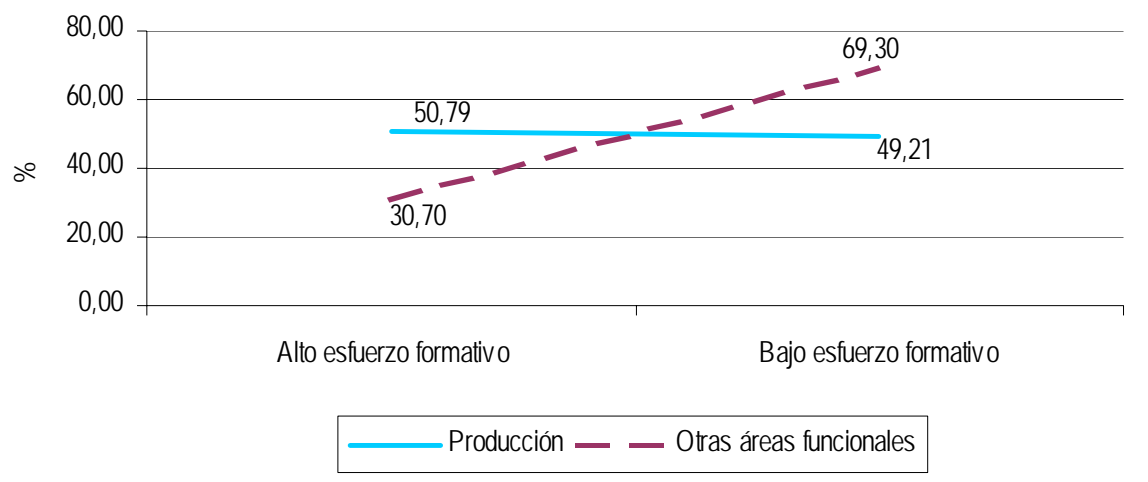

Base: Producción: $n=63$; Otras áreas funcionales: $n=228$

Figura 3. Relación entre área funcional en la que se desarrolla la actividad laboral y esfuerzo formativo. Ocupados-Ocupados

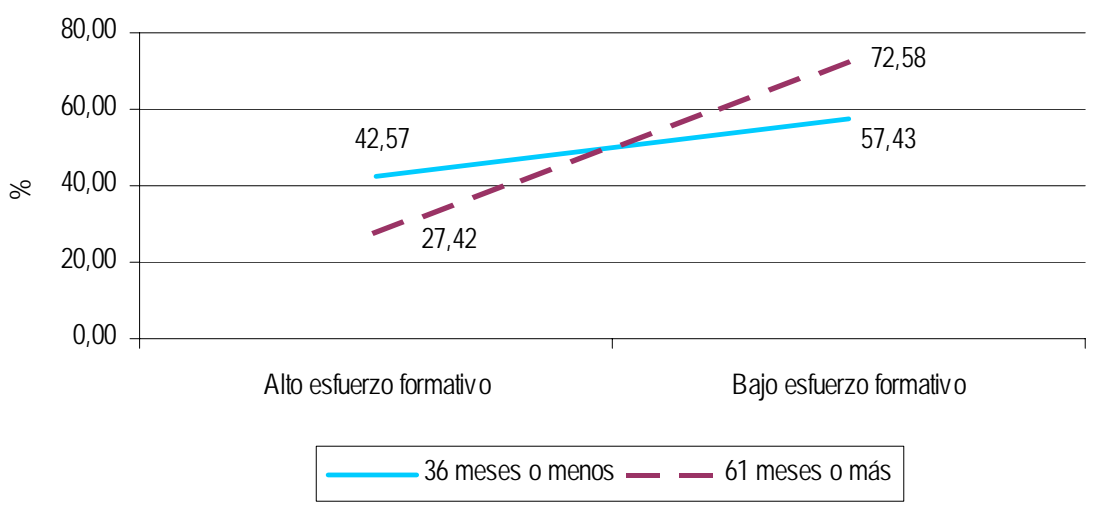

Base: 36 meses o menos: $\mathrm{n}=101 ; 61$ meses o más: $\mathrm{n}=124$

Figura 4. Relación entre tiempo ocupado y esfuerzo formativo.Ocupados-Ocupados

En la categoría de ocupados que pasan a desocupados, no se hallan factores asociados al alto esfuerzo formativo, tal y como acabamos de ver en los trabajadores que mantienen el empleo. De manera concreta, y por contraste con el resultado anterior, en el caso de los trabajadores que pierden su empleo, el esfuerzo formativo no se halla asociado a la función productiva o no productiva, y tam- poco al hecho de llevar más o menos tiempo ocupado.

Variación de la situación laboral de los desocupados

Como resultado, el esfuerzo formativo no se asocia a la variación de la situación laboral de los desocupados. Entre los que se encuentran desocupados, en el momento de finalizar la última acción formativa, en torno 
al $40 \%$ pasan a estar ocupados, independien-

(Figura 5).

temente del esfuerzo formativo realizado

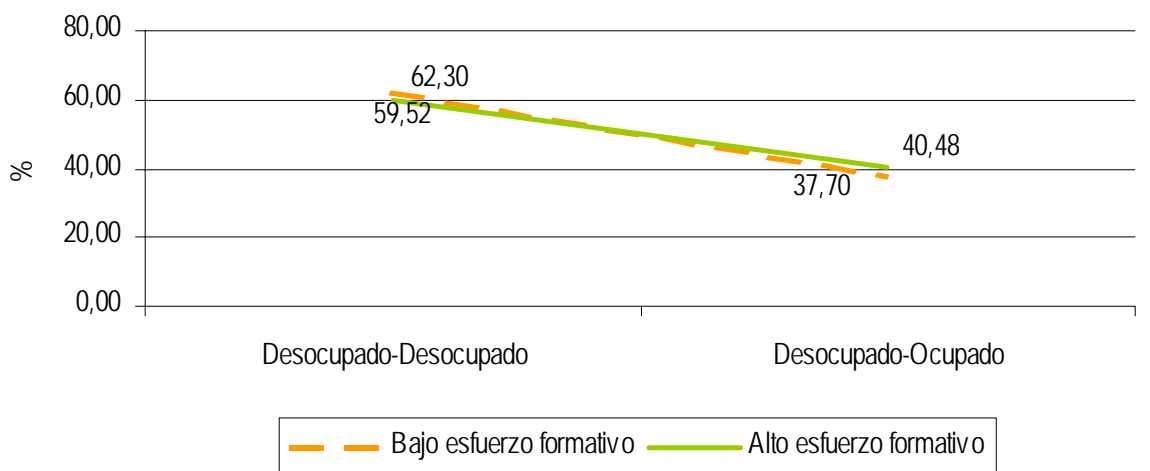

Base: Desocupado-desocupado: $\mathrm{n}=63$; Desocupado-ocupado: $\mathrm{n}=40$

Figura 5. Relación entre esfuerzo formativo y variación de la situación laboral de desocupados

\section{Dimensión organizacional: Mejora del trabajo}

Este es el ámbito en el que el esfuerzo formativo tiene una mayor repercusión. Se encontraron asociaciones positivas y estadísticamente significativas entre el esfuerzo formativo y ambas variables de mejora o enriquecimiento del trabajo: variación de categoría profesional (ascenso o promoción) y variación de funciones/responsabilidades.

\section{Variación de categoría profesional}

Los trabajadores que han realizado un alto esfuerzo formativo, con mayor frecuencia que aquellos que realizan un bajo esfuerzo, ascienden de categoría profesional (Chicuadrado=6,078; $p=0,014)$. El $65 \%$ de los que realizan un alto esfuerzo formativo ascienden frente a un $35 \%$ en el caso de los que realizan un bajo esfuerzo formativo (Figura 6).

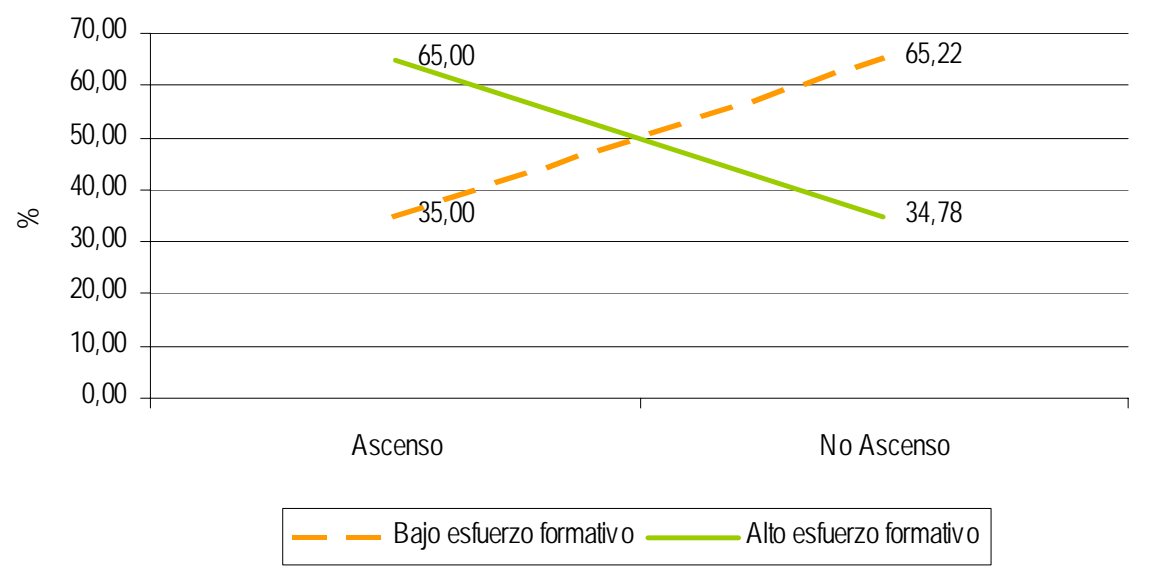

Base: Ascenso: $\mathrm{n}=20$; No ascenso: $\mathrm{n}=276$

Figura 6. Relación entre esfuerzo formativo y variación de categoría profesional

Esta asociación se encuentra modulada por las siguientes variables: tamaño de empresa $\mathrm{y}$ cualificación profesional que se tiene. La asociación se mantiene en el caso de pequeñas/medianas empresas (Chi-cuadrado= $6,469 ; p=0,011)$, específicamente en empre- sas de 1 a 50 trabajadores (Chi-cuadrado= $6,116 ; \mathrm{p}=0,013)$ y entre trabajadores técni$\cos /$ cualificados $($ Chi-cuadrado $=8,241 ; \mathrm{p}=$ $0,004)$.

Los resultados muestran que en empresas con menos de 250 trabajadores quienes han 
realizado un alto esfuerzo formativo tienen 10,783 veces más posibilidades de haber ascendido que quienes han realizado un esfuerzo formativo bajo (Risk estimates $=10,783)$. Y en el caso de trabajadores en puestos de técnicos o trabajadores cualificados, la probabilidad de ascenso aumenta 4,863 veces (Risk estimates $=4,863$ ). Ver Figura 7.

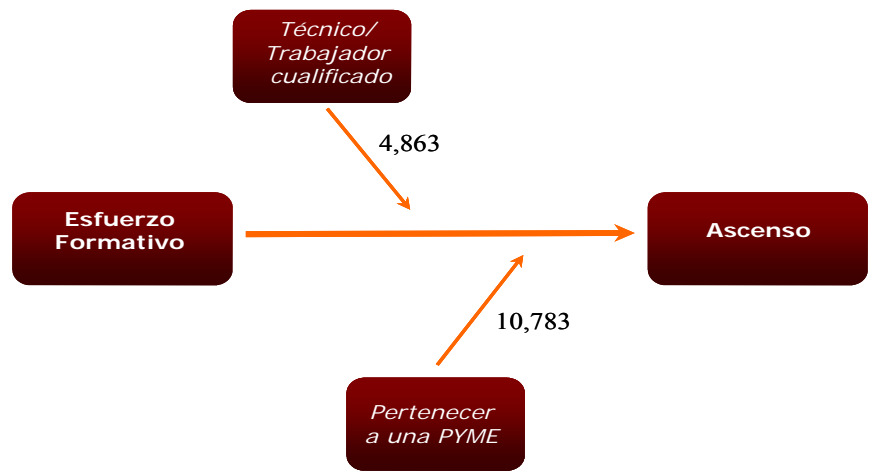

Figura 7. Variables moduladoras entre esfuerzo formativo y ascenso

Variación de funciones y/o responsabilidades

La variación de funciones y/o responsabilidades sigue un patrón similar al de la variación de la categoría profesional. Quienes realizaron un alto esfuerzo formativo, con mayor frecuencia que aquellos que realizaron un esfuerzo formativo bajo, cambian de funciones y/o responsabilidades (Chi-cuadrado $=5,115 ; \mathrm{p}=0,024)$. Ver Figura 8 .

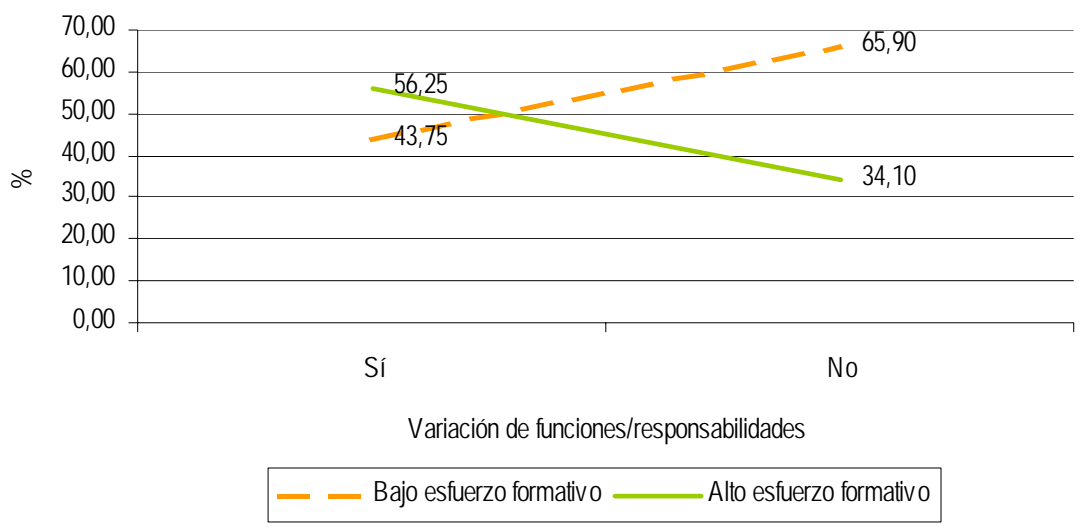

Base: Sí: $n=32 ;$ No: $n=261$

Figura 8. Relación entre esfuerzo formativo y variación de funciones/responsabilidades en el puesto de trabajo

Las variables que modulan esta relación, igual que en la variación de la categoría profesional, vuelven a ser: tamaño de empresa y cualificación profesional que se tiene. E igual que en el caso anterior, la asociación se establece en el grupo de pequeñas/medianas empresas (Chi-cuadrado=7,399; $p=0,007$ ), específicamente en empresas de 1 a 50 trabajadores (Chi-cuadrado $=11,573 ; \mathrm{p}=0,001)$ y en el grupo de trabajadores técni- $\cos /$ cualificados $($ Chi-cuadrado $=8,139 ; \mathrm{p}=$ $0,004)$.

Los resultados muestran que en empresas con menos de 250 trabajadores quienes han realizado un alto esfuerzo formativo tienen 3,845 veces más posibilidades de haber cambiado de funciones y/o responsabilidades que quienes han realizado un esfuerzo formativo bajo (Risk estimates $=3,845$ ). $Y$ en el caso de trabajadores en puestos de técnicos o traba- 
jadores cualificados, la probabilidad de cambio de funciones $\mathrm{y} / \mathrm{o}$ responsabilidades au- menta 4,863 veces (Risk estimates $=3,108$ ). (Figura 9).

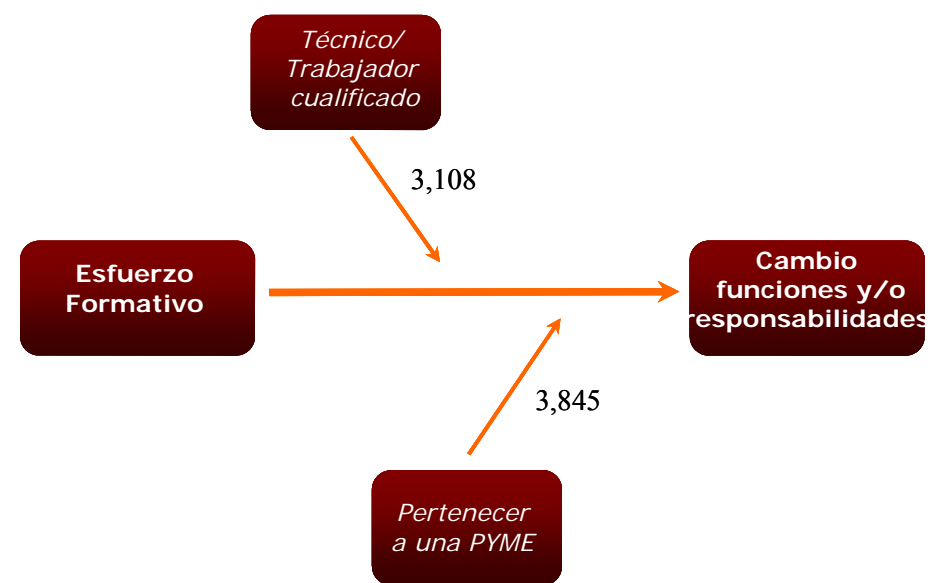

Figura 9. Variables moduladoras entre esfuerzo formativo y cambio de funciones/responsabilidades

Dimensión personal: Mejora en el nivel de desempeño laboral

Previamente a la exposición de los resultados, en éste apartado conviene recordar que para obtener una información más fiable de la medida del desempeño, únicamente se incluyeron los datos que proporcionaron aquellos trabajadores que manifestaron que en sus empresas disponían de sistemas formales de evaluación del desempeño. Como consecuencia, los resultados no confirman que un alto esfuerzo formativo se asocie a una mejora del desempeño. Por tanto, y de manera global o agregada, la variación en el desempeño laboral es independiente del esfuerzo formativo realizado.
Sin embargo, esta asociación se encuentra modulada por la variable "tamaño de empresa". Cuando la empresa es pequeña/mediana el esfuerzo formativo sí muestra un asociación significativa con la mejora del desempeño laboral (Chi-cuadrado $=6,979 ; \mathrm{p}=$ $0,008)$. En este tipo de empresas, el alto esfuerzo formativo, con mayor frecuencia se asocia a una mejora en el desempeño laboral. El $65,38 \%$ de los trabajadores de empresas pequeñas/medianas que realizan un alto esfuerzo formativo mejoran su desempeño respecto a un $34,62 \%$ en el caso de aquellos que realizan un esfuerzo formativo bajo (Figura 10).

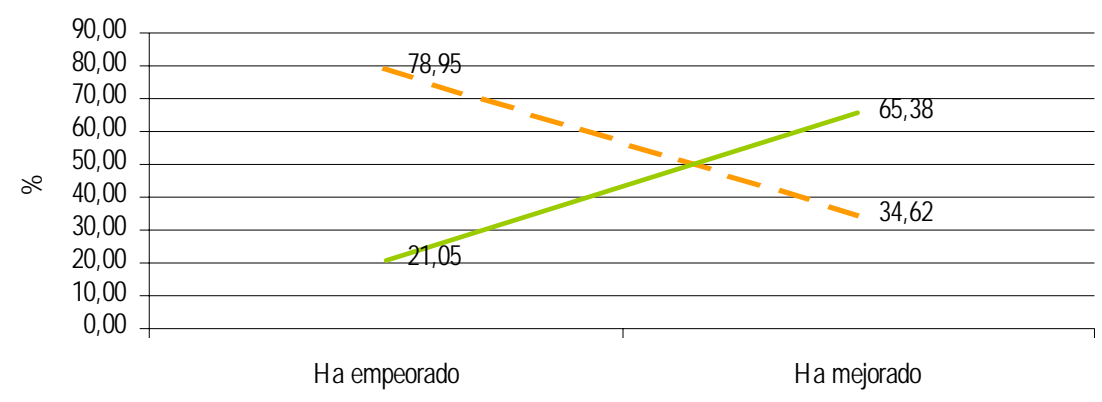

Variación del Desempeño Laboral

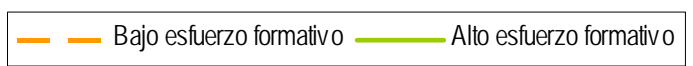

Base: Ha empeorado: $\mathrm{n}=19$; Ha mejorado: $\mathrm{n}=26$

Figura 10. Relación entre esfuerzo formativo y variación en desempeño laboral. Empresas pequeñas/medianas 
En empresas con menos de 250 trabajadores quienes han realizado un alto esfuerzo formativo tienen 0,463 veces más posibilidades de haber mejorado el desempeño laboral que aquellos que han realizado un esfuerzo formativo bajo (Risk estimates $=0,463)$ (Figura 11).

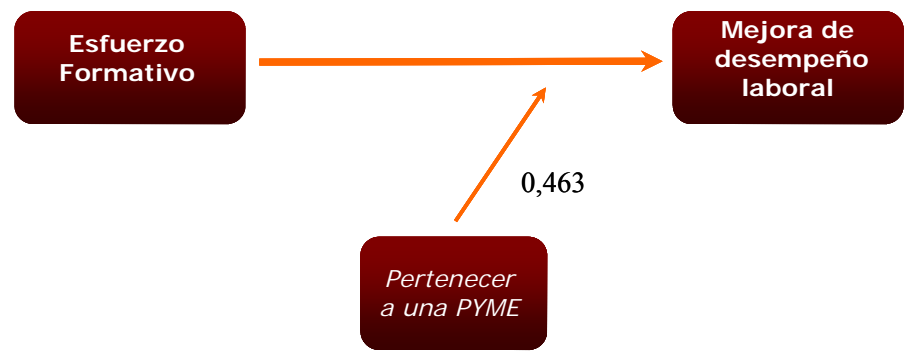

Figura 11. Variables moduladoras entre esfuerzo formativo y desempeño

\section{Conclusiones y discusión de resultados}

En general, la asociación entre esfuerzo formativo o cantidad de formación y empleabilidad arroja resultados dispares dependiendo de qué indicador de empleabilidad se adopte a la hora de buscar una asociación. Por tanto, globalmente, la hipótesis de partida sólo se confirma parcialmente. En primer lugar, la asociación más clara queda establecida en el grupo de trabajadores ocupados. En ellos, el esfuerzo formativo realizado contribuye a que asciendan o cambien sus funciones. Por consiguiente, en referencia a la dimensión organizacional de la empleabilidad, la hipótesis específicamente formulada se confirma. Sin embargo, al estudiar la asociación entre esfuerzo formativo y mantenimiento/consecución del empleo (en trabajadores ocupados y desempleados respectivamente), los datos no arrojan ninguna asociación significativa. En consecuencia, en el caso de la dimensión socioeconómica de la empleabilidad, la hipótesis queda claramente refutada.

Dado este resultado, cabe matizar que el mantenimiento o la consecución del empleo dependa en mucha mayor medida del entorno económico que de la propia formación. Sin embargo, tampoco se pueden excluir como causa otras variables que podrían derivarse de la gestión realizada por las propias empresas, o incluso de la concepción y fun- cionamiento del modelo público de gestión de la formación vigente. Sobre todo si se tiene en cuenta que otros estudios en otros contextos aportan evidencias de la existencia de una relación positiva entre formación y empleo. A ello cabe añadir que en nuestro propio estudio, la influencia del esfuerzo formativo en el desempeño individual tampoco queda demostrada de una manera tan contundente como en un principio cabría esperar. Así, en el caso del efecto de la formación en la dimensión individual/personal de la empleabilidad, la hipótesis de partida solo se confirma parcialmente. En efecto, únicamente cuando los trabajadores pertenecen a una pyme o micro pyme, la asociación entre esfuerzo formativo y desempeño laboral se establece como positiva y significativa. En este sentido, podemos decir que los datos muestran que el menor tamaño de la empresa determina un mejor retorno social de la cantidad de formación realizada. Una relación que también confirman otros estudios como se verá a continuación.

\section{Variables de esfuerzo formativo pre- dictoras de la empleabilidad}

El número de acciones formativas es mejor predictor del enriquecimiento de la actividad $\mathrm{o}$ rol (promoción y cambio de funciones/responsabilidades) que el número de horas de formación. Sin embargo, el número de horas es un criterio fundamental en la planificación y gestión formativa. Los moti- 
vos de este resultado precisan de investigación adicional utilizando técnicas cualitativas (entrevistas en profundidad, técnicas grupales...) que aporten información por parte de empresarios, mandos, directivos, o específicamente, profesionales de la gestión de los recursos humanos, acerca de los motivos que les llevan a utilizar un criterio u otro en el momento de realizar promociones o adoptar decisiones de movilidad funcional en los trabajadores.

Podemos anticipar una primera hipótesis considerando que esto puede ser debido a que a la hora de realizar una promoción o un cambio de funciones/responsabilidades, en un primer momento, lo más relevante consiste en aportar alguna prueba de conocimiento, aunque no sea exhaustivo. Se trataría de un ejercicio de acreditación informal donde se valora el hecho de haber realizado alguna formación, pero no tanto la duración de la misma.

\section{Consecución y mantenimiento del em- pleo (dimensión socioeconómica)}

Los resultados del presente estudio en cuanto a consecución de empleo no confirman los hallazgos de otros estudios en los que se utilizó como variable independiente la realización o no de formación (Nivorozhkin y Nivorozhkin, 2005; Boon Heng et al. 2006). En nuestro caso, no se observaron diferencias en esfuerzo formativo entre aquellos desocupados que se mantuvieron desocupados y los que encontraron empleo desde la finalización de la última acción formativa. Si bien es cierto que en los estudios señalados, al contrario del presente, se presentan los resultados en función de variables sociodemográficas: varones y trabajadores temporales o a tiempo parcial (Boon Heng et al. 2006) y trabajadores poco cualificados (Nivorozhkin y Nivorozhkin, 2005), con mayor frecuencia sus sujetos consiguen un nuevo trabajo después del tratamiento formativo. Desde otra perspectiva, Mamaqi y Miguel (2009), logran evidencia empírica de que la formación continua específica, concer- tada entre empleado y empleador y a niveles superiores al básico, logra incrementar la estabilidad laboral.

Sin embargo, al margen de la segmentación de los participantes en acciones formativas en función de variables sociodemográficas (a tener en cuenta en futuros análisis), el hecho de que el esfuerzo formativo no aparezca asociado con la consecución de un nuevo empleo, nos remite al importante peso que en esta relación puede estar teniendo el momento de recesión económica en que se realizó el estudio. La realización de estudios de similares características donde se recoja la relación formación-consecución de la ocupación en diferentes contextos macroeconómicos permitirá conocer mejor cómo afecta este factor a la relación entre las variables objeto del estudio. A pesar de todo, dentro del modelo de gestión vigente en España, uno de los objetivos capitales de la formación consiste en la consecución del empleo. Sin embargo, la aplicación del modelo actual no parece estar logrando este objetivo. Quizás, en alguna medida, debido a una predominancia de la operativa orientada al alcance, por encima de la orientación al resultado (FEMP, 2009). Algo en lo cual habría que indagar más.

Respecto al mantenimiento de la ocupación, tampoco se halla asociación con el esfuerzo formativo. Quizás por los mismo motivos apuntados en el párrafo anterior. Aun con todo, en este colectivo de trabajadores que mantienen su empleo, realizan un esfuerzo formativo mayor quienes se encuentran en un área funcional de producción. Este hallazgo es coherente con los estudios ligados a la crisis económica de principios de los años 90. En ellos se comprueba cómo la producción industrial es el sector que en mayor medida mantiene la inversión en formación (Ramírez del Río, A. 1997). En una situación económica de crisis, la rentabilidad social de la formación sería mayor en áreas funcionales y sectores económicos vinculados directamente con la producción ya que son éstas 
áreas y sectores donde el resultado económico es más fácil de ver.

En este mismo grupo de participantes que mantienen la ocupación, realizaron también un esfuerzo formativo mayor los que llevaban menos tiempo en su puesto de trabajo (36 meses o menos frente a 61 meses o más). Este comportamiento podría poner de relieve una actitud de interés de empresas y trabajadores en adquirir los conocimientos necesarios para desarrollar con un buen desempeño su puesto de trabajo. Este resultado estaría en línea con la valoración que los trabajadores hacen de la formación (CEOE, 2001, citado por Pineda, 2007). El 90\% de ellos la valoran como un medio para la superación y el desarrollo personal y para alcanzar nuevas competencias profesionales y un $25 \%$ la valora como un recurso para encontrar trabajo en otra empresa.

Con todo, lejos de confirmarse una asociación positiva entre esfuerzo formativo $y$ mantenimiento de la ocupación se observa una llamativa tendencia inversa (a mayor formación menor mantenimiento del empleo) que no obstante, no resulta estadísticamente significativa. Es posible que ante la expectativa de perder el puesto de trabajo, este grupo se procurase una mayor preparación profesional, bien para reducir la posibilidad de hallarse en una situación de inactividad, bien para contar con más recursos personales que faciliten o incrementen la posibilidad de obtener un nuevo empleo. Ello nos lleva a plantear que, estando ocupados, la expectativa del desempleo podría ser un potente impulsor del mayor esfuerzo formativo. No obstante, esta sería una hipótesis a comprobar en futuros estudios.

\section{Enriquecimiento de la actividad (di- mensión organizacional)}

En lo que se refiere a la mejora de la posición del trabajador en la organización o enriquecimiento de su actividad/rol (mejora de la categoría profesional y cambio de funciones/responsabilidades), ésta aparece favora- blemente asociada al esfuerzo formativo. Por tanto, confirmamos que la mayor participación formativa tiene un efecto positivo en la mejora de la posición formal o informal del trabajador. Sobre todo si tenemos en cuenta que las empresas pueden hacer planes y programas especiales de desarrollo para aquellas personas consideradas como potencialmente válidas para la promoción. Sin embargo, en la mejora de la posición del trabajador también puede influir la movilidad funcional que conlleva la propia dinámica de expansión o contracción de las organizaciones. Por ejemplo, en una situación de restricción económica, la salida de un trabajador puede no reponerse y entrañar mayores responsabilidades para quien permanece ocupado en la misma empresa. En estos casos, aquellos trabajadores que realizan un esfuerzo formativo mayor, tienen también mayores posibilidades de promocionar o cambiar de funciones y/o responsabilidades.

Esta mejora de la actividad del trabajador como consecuencia del esfuerzo formativo se pone de manifiesto en PYME (250 trabajadores o menos) y fundamentalmente en micro PYME (entre 1 y 50 trabajadores). Se trata de un resultado coherente con lo encontrado por Yli-Renko et al. (2001) y Autio et al. (2000) quienes expresan que el tamaño de la organización puede influir en la adquisición y explotación del conocimiento. Las empresas de gran tamaño para alcanzar ventaja competitiva cuentan con otros recursos además del conocimiento (Gopalakrishnan y Bierly, 2006). Pueden acceder a personal especializado, equipos, software, procedimientos y otras herramientas para responder a demandas específicas del entorno (YliRenko et al., 2001; Debowski, 2006). Por otra parte su alta estructuración, con políticas de recursos humanos muy formalizadas $y$ complejas (gestión del talento, sistemas de evaluación $\left.360^{\circ} \ldots\right)$ puede determinar que su reactividad a la formación sea más baja. La movilidad del trabajador en estas empresas (ascenso o promoción y cambio de funciones/responsabilidades) no es una consecuen- 
cia directa ni inmediata de la formación recibida, sino que posiblemente esta sujeta más procesos estandarizados.

Las pymes, por el contrario, han de responder en mayor medida a las demandas del mercado con el personal con el que cuentan. En general disponen de menos recursos, con lo que tienen menos posibilidades de recurrir a personal especializado. De este modo, ampliar la polivalencia de sus trabajadores actuales se convierte en un elemento estratégico. Por otra parte, su baja estructuración les permite mayor flexibilidad y agilidad para responder a los cambios (Bierly y Daly, 2002; Gopalakrishnan y Bierly, 2006) tanto externos como internos. Por tanto, en las empresas pequeñas, la ampliación de conocimientos o la mayor especialización del personal mediante la formación tienen un efecto más inmediato en la movilidad interna que en las grandes.

Por otra parte, de acuerdo con lo que se encontró entre los trabajadores que se mantienen ocupados, los que pertenecen al área de producción son el colectivo en el que el esfuerzo formativo aparece más asociado a la mejora de su posición profesional. Este dato es consistente con lo hallado por Krueger y Rouse (1998) al estudiar el efecto de la formación en el trabajo realizado, según diferentes tipos de empresa. Sus resultados mostraron que en las empresas de manufactura la formación tuvo unos efectos positivos para los trabajadores, efectos que no se hallaron en las empresas de servicios.

De nuevo, estas conclusiones pueden venir determinadas por el hecho de que sean puestos más directamente relacionados con el output del negocio. Al estar las necesidades de estos puestos más directamente vinculadas al resultado empresarial, la formación cobra una mayor importancia para las organizaciones. Cabe por tanto que en tiempos de crisis la formación que más directamente impacta en los resultados económicos de la empresa no se reduzca, o no se reduzca tan- to, al contrario que la formación con un resultado más indirecto.

Finalmente, en el caso de los trabajadores técnicos/trabajadores cualificados, también hallamos una mayor incidencia de la formación en la promoción o cambio de funciones. Al contrario que en el trabajo de Nivorozhkin y Nivorozhkin (2005), donde se hallan evidencias de que la formación mejora el desarrollo profesional de los trabajadores operarios, nuestro estudio no establece esta última asociación de manera específica. La explicación podría ser similar al caso anterior referido al área productiva. Cabe esperar un mayor valor obtenido del trabajo de los técnicos/trabajadores cualificados y consiguientemente, en un momento de restricciones económicas, el esfuerzo formativo tendría más sentido hacerlo en el colectivo que mayor valor aporta. La formación para este grupo de trabajadores, por tanto, pasaría a ser un factor de gran relevancia por contribuir de forma diferencial al mantenimiento o mejora de los resultados globales de la empresa.

\section{Desempeño laboral (dimensión indivi- dual)}

El resultado positivo de la formación en el desempeño laboral aparece de forma consistente en diferentes estudios (Bartel, 1995; Boon Heng, et al. 2006). Sin embargo, en nuestro análisis, por lo general, la variación en el desempeño laboral es independiente del esfuerzo formativo realizado. No obstante, al restringir el análisis a lo que ocurre en las pequeñas/medianas empresas (250 trabajadores o menos) se observa que el mayor esfuerzo formativo tiene un mejor resultado. De manera consistente con el dato anterior referido a la dimensión organizativa de la empleabilidad, ello podría significar que las empresas menores aprovechan mejor la formación en términos de su aplicación al trabajo. Quizás por ser más pequeñas y sensibles a los costes se encuentran más orientadas a rentabilizar las ausencias del puesto de trabajo debidas a la asistencia a formación. Asimismo, también es posible que teniendo un 
menor nivel de estructuración y/o procedimentación que las empresas grandes, sean más permeables a la aportación individual.

\section{Propuestas de futuro}

Este análisis es una primera aproximación a la relación esfuerzo formativoempleabilidad. Estudios posteriores en esta misma línea permitirán profundizar y conocer mejor los factores que inciden en dicha relación. En este sentido, algunas sugerencias serían las siguientes:

- La replicación de estudios similares al realizado con otros planes formativos y asociaciones promotoras de formación aportará solidez a los resultados obtenidos. Por ejemplo, contrastar la nula asociación entre esfuerzo formativo y consecución/mantenimiento del empleo es de gran importancia, no solamente por el interés científico, sino por el posible cuestionamiento o necesidad de revisión de las políticas de empleo y modelo formativo vigentes a que podría llevar un resultado como este.

- Complementariamente, cabe plantearse la influencia de otras variables que no han sido analizadas. Nos referimos a las que son específicas de la formación (tipo de contenidos, modalidades de impartición, recursos tecnológico-colaborativos, etc.), las sociodemográficas (sexo, edad, nivel académico, etc.) y las de contexto macroeconómico (expansión o constricción económica, renta per capita zonal, predominancia de sectores productivos, etc.). Además, también conviene incluir el factor tiempo como variable independiente asociada al efecto de la formación.

- Desde una perspectiva metodológica, la inclusión de un grupo control conferiría una mayor robustez a los resultados obtenidos. Además, la realización de estudios longitudinales permitiría mayor información sobre el efecto de la variable tiempo en la relación esfuerzo formativoempleabilidad.
- El trabajo realizado sugiere que la expectativa del desempleo podría ser un importante impulsor de la realización de formación. Un mayor conocimiento acerca del papel que la expectativa del desempleo puede tener en la decisión de los trabajadores para emprender iniciativas de formación sería útil para hacer políticas adecuadas de formación y empleo.

- En los análisis practicados, el número de horas de formación no resultó ser un buen predictor en las decisiones de movilidad funcional de los trabajadores. La realización de estudios cualitativos aportaría información relevante acerca del valor real que las empresas otorgan al número de acciones formativas y al número de horas de formación en el momento de promocionar o cambiar de funciones a los trabajadores. Como se ha indicado, el número de horas es un criterio ampliamente difundido en la gestión de la formación para "dimensionar" la formación o el esfuerzo formativo que se realiza.

\section{Referencias}

Arulampalam, W.; Booth, A \& Bryan, M.L. (2004). Training in Europe. Journal of the European Economic Association, 2(3), 346360.

Autio, E.; Sapienza, H.J. \& Almeida, J.G. (2000). Effects of Age at Entry, Knowledge Intensity, and Imitability on International Growth. Academy of Management Journal, 43(5), 909-924.

Bartel, A. (1995). Training, Wage Growth, and Job Performance: Evidence from a Company database. Journal of Labor Economics, 13(3), 401-25.

Barrett, A. \& O'Connell, P. (2001). Does Training Generally Work? The Returns to In-Company Training. Industrial and Labor Relations Review, 54(3), 647-62.

Bierly, P. \& Daly, P. (2002). Aligning Human Resource Management Practices and Knowledge Strategies: A Theoretical Framework. En C.W. Choo y N. Bontis (Ed.), The Strategic Management of Intel- 
lectual Capital and Organizational Knowledge (pp. 277-295). New York: Oxford University Press).

Boon Heng, A.; Cheolsung, P.; Haoming, L.Thangavelu, S.M. and Wong, J. (2006). The impact of structured training on worker's employability and productivity. SCAPE Working Paper Series, 2007/02). Consultado el 20 de agosto de 2011 en: http://www.mom.gov.sg/Publications/mrsd The Impact of Structured Training.pdf

Budria, S. \& Pereira, P. (2004). On the returns to training in Portugal. IZA Discussion Paper, 1429.

Cassidy, M.; Görg, H. \& Strobl, E. (2005). Knowledge accumulation and productivity: Evidence from plant level data for Ireland. Scottish Journal of Political Economy, 52, 344-358.

CEOE (2001). La formación en las empresas españolas y su relación con el empleo. Estudio 2000-2001.

Colton, T. (1979). Estadística en medicina (pp. 185). Barcelona: Salvat.

Dearden, L.; Reed, H. \& Van Reenen, J. (2000). Who Gains When Workers Train? Training and Corporate Productivity in a Panel of British Industries. IFS Working Paper, 00/04.

Debowski, S. (2006). Knowledge Management. Brisbane: John Wiley \& Sons.

Domènech Massons, J.M. (1995). Comparación de dos proporciones. Medidas de riesgo. En Métodos estadísticos en ciencias de la salud. Barcelona: Signo.

Domènech Massons, J.M. (1995). Relación entre variables categóricas. Pruebas de Chi-cuadrado. En Métodos estadísticos en ciencias de la salud. Barcelona: Signo.

FEMP (2009). Griker Orgemer - Ramírez del Río, A. (Col.). Formación continua y modernización de la Administración Local: El reto de la evaluación del impacto. Consultado el 22 octubre de 2011 en: http://www.femp.es/files/11-593-

fichero/Formaci $\% \mathrm{C} 3 \% \mathrm{~B} 3 \mathrm{n} \% 20$ Continua $\% 20 \mathrm{y} \%$ 20Modernizaci $\%$ C3\%B3n $\% 20$ de $\% 201 \mathrm{a} \% 20$ Administra- ci $\% \mathrm{C} 3 \% \mathrm{~B} 3 \mathrm{n} \% 20 \mathrm{Local} \% 20 \mathrm{El} \% 20$ reto $\% 20$ de $\% 201 \mathrm{a} \% 20$ evaluaci $\% \mathrm{C} 3 \% \mathrm{~B} 3 \mathrm{n} \% 20 \mathrm{del} \% 2$ 0impacto.pdf.

Finn, D. (2000). From full employment to employability: a new deal for Britain's unemployed? International Journal of Manpower, 21(5), 384-389.

Hillage, J. \& Pollard, E. (1998). Employability: Development a framework for policy análisis. Research Brief, 85, 1-4.

Greenberg, D.; Michalopoulos, C. \& Robins, P.K. (2003). A meta-analysis of government-sponsored training programs. Industrial and Labor Relations Review, 57, 3153.

Gopalakrishnan, S.; Bierly, P. (2006). The Impact of Firm Size and Age on Knowledge Strategies During Product Development: A Study of the Drug Delivery Industry, IEEE Transactions on Engineering Management, 53(1): 3-16.

Hempell, T. (2005). What's Spurious? What's Real? Measuring the Productivity Impacts of ICT at the Firm Level. Empirical Economics 30(2), 427-464.

Herrarte, A y Sáez, F. (2004). Formaciónempleo: una evaluación. Cuadernos de Economía, 27, 147-174.

Knight, P. \& Yorke, M. (2003). Assessment, Learning and Employability. Glasgow: Society for Research into Higher Education and Open University Press.

Krueger, A. \& Rouse, C. (1998). The effects of workplace education on earnings, turnover and job performance. Journal of Labor Economics, 16, 61-94.

Kuckulenz, A. \& Zwick, T. (2003). The Impact of Training on Earnings: Differences Between Participant Groups and Training Forms, ZEW Discussion Papers, 3, 57.

Kuhn, T.S. (1962). La estructura de las revoluciones científicas. Madrid: Fondo de Cultura Económica.

Mamaqi, X y Miguel, J.A. (2009). Impacto de la formación continua sobre la estabilidad laboral. En Actas del XIII Congreso de Economía Laboral, Julio, Zaragoza, España. Consultado el 8 de octubre de 2011 en: http://www.unizar.es/centros/fccee/doc/jorn 
adas economia/ponencias/1_1_Mamaqi-

Miguel.pdf

Mamaqi, Xhevrie \& Miguel, Jesús A. (2011). The professional profile of trainers working in continuous training in Spain. RELIEVE, v. 17, n. 1, art. 2. Consultado el 15 de abril de 2011 en: http://www.uv.es/RELIEVE/v17n1/RELIE VEv17n1 2eng.htm.

Molina, J. \& Ortega, R. (2003). Effects of employee training on the performance of North-American firms. Applied Economics Letter, 10, 549-552.

Nivorozhkin, A. and Nivorozhkin, E. (2005). Do Government Sponsored Vocational Training Programs Help the Unemployed Find Jobs? Evidence from Russia. Applied Economics Letters, 14 (1-3):5-10.

Pérez R., E. y Malvezzi, S. (2008). Empleabilidad, cambios y exigencias psicosociales en el trabajo. Universitas Psycológica, 7(2), 319-334.

Pineda, P. y Sarramona, J. (2006). El nuevo modelo de formación continua en España: balance de un año de cambios. Revista de Educación, 341, 705-736.

Pineda Herrero, Pilar (2007). La formación continua en España: balance y retos de futuro RELIEVE, v. 13, n. 1. http://www.uv.es/RELIEVE/v13n1/RELIE VEv13n1_2.htm. Consultado el 5 febrero 2010.

Puhani, P.A. (2002). Advantage through Training? A Microeconometric Evaluation of the Employment Effects of Training and Job Subsidy Programmes. Labour, 16, 569608.

Ramírez del Río, A. (1997). Valoración de la Formación. Cómo rentabilizar los costes de formación. Madrid: Griker \& Asociados.

Regner, H. (2002). The effects of on-the-job training on wages in Sweden. International Journal of Manpower, (4)23, 326-344.

Rentaría, P., E. y Malvezzi, S. (2008). Empleabilidad, cambios y exigencias psicosociales en el trabajo. Universitas Psycológica, 7(2), 319-334.

Sáez y Torres. (1999). Encuentro sobre Empleabilidad de FUNDIPE. Consultado el 23 de abril de 2011 en http://www.fundipe.es/archives/INFORME E Seguro.pdf

Van de Wiele, P. (2010). The impact of training participation and training costs on firm productivity in Belgium. International Journal of Human Resource Management, 21(4), 582-599.

Yli-Renko, H.; Autio, E.; \& Sapienza, H.J. (2001). Social Capital, Knowledge Acquisition, and Knowledge Explotation in Young Technology-Based Firms. Strategic Management Journal, 22 (6-7), 587-613.

Zwick, T. (2004). Employee Participation and Productivity. Labour Economics, 11(6),715-740.

Zwick, T. (2006). The impact of training intensity on establishment productivity. Industrial Relations, 45(1), 26-46. 


\section{ANEXO}

\section{CUESTIONARIO SOBRE FORMACIÓN Y EMPLEABILIDAD}

(Se han eliminado elementos gráficos y textos identificativos de la entidad promotora del plan de formación estudiado)

\section{Código Cuestionario}

\section{DATOS IDENTIFICATIVOS DEL PARTICIPANTE (Rellena encuestador)}

Nombre y Apellidos

Número de teléfono

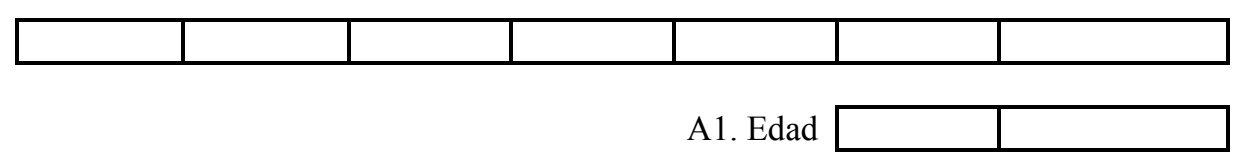

A2. Sexo

1.Mujer

2.Varón

A7. Categoría profesional/Cargo que ocupa dentro de la empresa

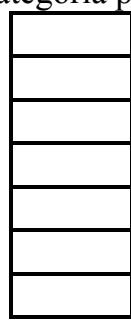

1.Directivo

2.Mando medio

3.Técnico

4.Trabajador cualificado

5.Trabajador No cualificado

98. Otro: ¿Cual?

99. $\mathrm{Ns} / \mathrm{Nc}$

\section{DATOS IDENTIFICATIVOS DE FORMACIÓN}

(Rellena encuestador en Cuadro Resumen Seguimiento de la Formación 1)

19. Número de acciones formativas o cursos en los que ha participado en el último año

Especificar Número/Cantidad

I7. Denominación de cada una de las acciones formativas

I10.Área temática de cada acción formativa (o curso)

I11. Número de horas de cada acción formativa

I8. Modalidad de cada una de las acciones formativas

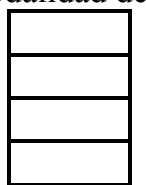

1.Presencial

2.Distancia

3.Mixta

4.Teleformación (Solo usa plataforma)

I12. Fecha de finalización de cada acción formativa 
Ramírez-del-Río, Antonio \& Garrido Casas, Jorgina (2011). Evaluación del impacto del esfuerzo formativo en la empleabilidad de los trabajadores en el contexto del modelo formativo tripartito español. RELIEVE, v. 17, n. 2 , art. 4.

http://www.uv.es/RELIEVE/v17n2/RELIEVEv17n2 4.htm

CUADRO RESUMEN-SEGUIMIENTO DE LA FORMACIÓN I

\begin{tabular}{|l|l|l|l|l|l|}
\hline ACCIÓN & DENOMINACIÓN & ÁREA & DÍAS & MODALIDAD & FECHA FIN \\
\hline Acción1 & & & & & \\
\hline Acción2 & & & & & \\
\hline Acción3 & & & & & \\
\hline Acción4 & & & & & \\
\hline Acción5 & & & & & \\
\hline Total & & & & & \\
\hline
\end{tabular}

III. IMPACTO DE LA FORMACIÓN EN PARTICIPANTES (Preguntas a realizar al participante)

Buenos días/tardes: Me llamo... (identificación personal e institucional del encuestador) y me pongo en contacto con usted porque estamos realizando una encuesta para valorar el impacto de la formación realizada a través del S.R.E. Los datos aportados en este cuestionario son confidenciales y serán utilizados únicamente con fines estadísticos.

F1. ¿Conoce la existencia de la formación subvencionada y gratuita?

1-Sí F1.1 ¿Cómo la conoce, a través de qué vías?

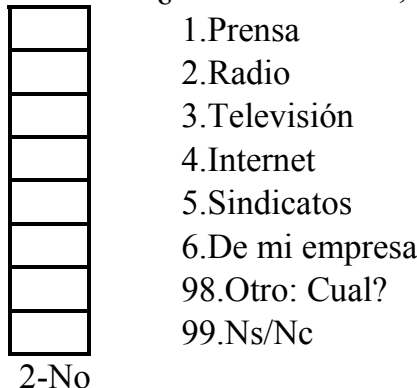

F2. ¿Recuerda hacer formación o algún curso en el ultimo año 2009?

1.Sí
2.Empecé un curso pero no lo terminé
3.No (: Explicar que sus datos se han obtenido del Anexo I que
firmó para el S.R.E. y cuya gestión hace xxxxxxx. Si no lo tiene claro pasar a P1)
4.Ns/Nc (: Explicar que sus datos se han obtenido del Anexo I
que firmó para el S.R.E. y cuya gestión hace xxxxxxx. Si no lo tiene claro pasar a P1)

F2.1 Hacer solo si F2=1 ó 2. ¿Recuerda si la formación era subvencionada?

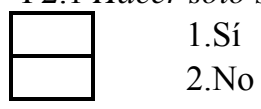

F2.2 Hacer solo si F2=1 ó 2. ¿Recuerda cuál era la entidad promotora?

1-Sí F2.2.1 ¿Cuál fue? Leer respuestas posibles

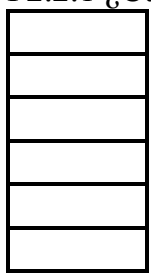

$$
\begin{aligned}
& \text { 1.xxxxxxxx } \\
& \text { 2.UGT } \\
& \text { 3.CCOO } \\
& \text { 4.CEIM-CEOE } \\
& \text { 98.Otro: Cual? } \\
& \text { 99.Ns/Nc }
\end{aligned}
$$

2-No Leer respuestas posibles. Si no recuerda pasar a F3

Ir a F2.3

F2.3 Hacer solo si F2.2.1=2,3,4 ó 98. No preguntar por la formación realizada con xxxxxxxx. ¿Qué acción/es formativas realizó con cada una de éstas entidades? Encuestador a rellenar en el Cuadro Resumen Seguimiento de la Formación II.

F2.4 Hacer solo si F2.2.1=2,3,4 ó 98. No preguntar por la formación realizada con xxxxxxxx. ¿Qué número de horas tenía cada acción formativa? Encuestador a rellenar en el Cuadro Resumen Seguimiento de la Formación II. 
F2.5 Hacer solo si F2.2.1=2,3,4 ó 98. No preguntar por la formación realizada con xxxxxxx. ¿En qué modalidad realizó cada una de las acciones formativas o cursos? Encuestador a rellenar en el Cuadro Resumen Seguimiento de la Formación II.

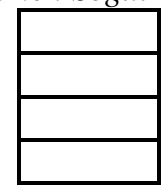

$$
\begin{aligned}
& \text { 1.Presencial } \\
& \text { 2.Distancia } \\
& \text { 3.Mixta } \\
& \text { 4.Teleformación (Solo usa plataforma en línea) }
\end{aligned}
$$

F2.6 Hacer solo si F2.2.1=2,3,4 ó 98. No preguntar por la formación realizada con xxxxxxx. ¿Aproximadamente cuándo terminó cada una de estas acciones formativas? Encuestador a rellenar en el Cuadro Resumen Seguimiento de la Formación II.

CUADRO RESUMEN-SEGUIMIENTO DE LA FORMACIÓN II

\begin{tabular}{|l|l|l|l|l|l|}
\hline ACCIÓN & ENTIDAD & DENOMINACIÓN & HORAS & MODALIDAD & FECHA FIN \\
\hline Acción1 & & & & & \\
\hline Acción2 & & & & & \\
\hline Acción3 & & & & & \\
\hline Acción4 & & & & & \\
\hline Acción5 & & & & & \\
\hline Total & & & & & \\
\hline
\end{tabular}

F3. Hacer a todos. ¿Terminó todas las acciones formativas que empezó? Si no recuerda todas en las que ha participado, enumerar un máximo de 5. Encuestador a rellenar en el Cuadro Resumen Seguimiento de la Formación III.

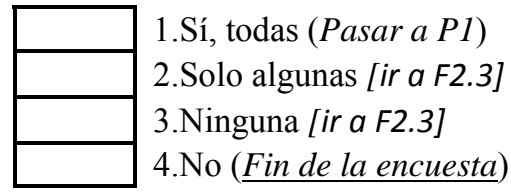

F4. Hacer solo si F3=2 y 3. ¿Por qué abandonó o no terminó la acción formativa? Preguntar por cada acción que haya abandonado. Respuesta múltiple. Encuestador a rellenar en el Cuadro Resumen Seguimiento de la Formación III.

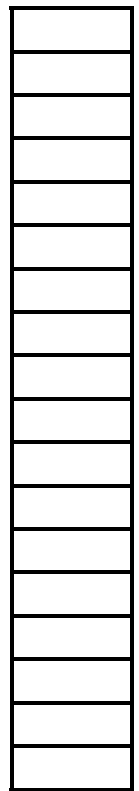

1.Horario

2.Material

3.Docente

4.Atención al cliente

5.Organización del curso

6.Instalaciones/equipamiento

7.Grupo-clase (heterogeneidad...)

8.Información previa al curso, comercialización

9.Expectativas del curso (no se han cumplido)

10.El nivel no era apropiado para usted (alto, bajo...)

11.Los contenidos no se ajustan al programa

12.Enfermedad

13.Fallecimiento de algún familiar

14.He encontrado trabajo

15.Por motivos de trabajo (carga de tiempo...)

16.Me voy/me echan de la empresa

98.Otro.¿Cual?

99. Ns/Nc

CUADRO RESUMEN-SEGUIMIENTO DE LA FORMACIÓN III

\begin{tabular}{|l|l|l|l}
\hline ACCIÓN & FINALIZADO & ABANDONO & MOTIVOS DE ABANDONO \\
\hline
\end{tabular}


Ramírez-del-Río, Antonio \& Garrido Casas, Jorgina (2011). Evaluación del impacto del esfuerzo formativo en la empleabilidad de los trabajadores en el contexto del modelo formativo tripartito español. RELIEVE, v. 17, n. 2, art. 4. http://www.uv.es/RELIEVE/v17n2/RELIEVEv17n2 4.htm

\begin{tabular}{|l|l|l|l|} 
Acción1 & & & \\
\hline Acción2 & & & \\
\hline Acción3 & & & \\
\hline Acción4 & & & \\
\hline Acción5 & & & \\
\hline Total & & & \\
\hline
\end{tabular}

P1. Hacer a todos. Actualmente, ¿cuál es su situación laboral? ¿Está usted ocupado/trabajando o está desempleado?

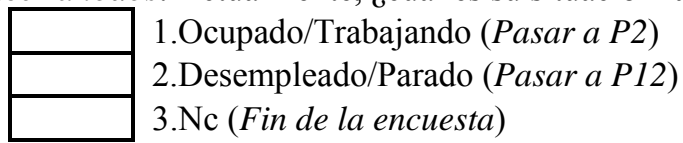

P2. ¿Desde cuándo lleva usted en esta situación laboral?, ¿Desde cuándo está usted ocupado?

Especifique cuánto tiempo

P3. Hacer solo si P1=1. ¿A qué área funcional/departamento pertenece usted dentro de su organización? Respuesta única, en caso de duda, leer respuestas.

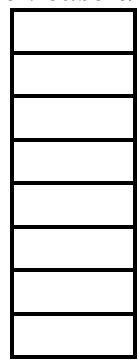

1.Dirección

2.Mantenimiento

3.Nc (Fin de la encuesta)

4.Producción

5.Comercial

6.Administración

98. Otro¿Cuál?

99.Ns/Nc

P4. Hacer solo si $P 1=1$. ¿Cuál es la actividad principal de la empresa en la que usted trabaja? ¿Cuál es su sector productivo? Respuesta única, en caso de duda, leer respuestas.

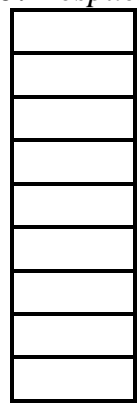

1.Ahorro

2.Artes gráficas

3.Banca

4.Medios de comunicación/actividades culturales o deportivas

5.Limpieza

6.Seguridad

7.Seguros, Oficinas y Despachos

98.Otro: ¿Cual?

99. Ns/Nc

P4.2 Hacer solo si $P 1=1$ ¿Qué puesto ocupa usted dentro de su empresa?

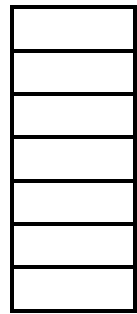

1.Directivo

2.Mando intermedio

3.Técnico

4.Puesto cualificado

5.Puesto no cualificado

98.Otro ¿Cuál?

99. Ns/Nc

P5. Hacer solo si P1=1. Tamaño de la empresa. ¿cuántos trabajadores aproximadamente forman la plantilla de su empresa?

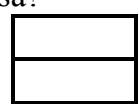

1.Entre 1 y 50 trabajadores

2.Entre 51 y 250 trabajadores 
3.Entre 251 y más trabajadores

P6. Hacer solo si P1=1. Desde la fecha X (encuestador tener en cuenta la fecha de finalización de la última acción formativa) ¿ha cambiado usted de empresa o sigue usted trabajando en la misma?

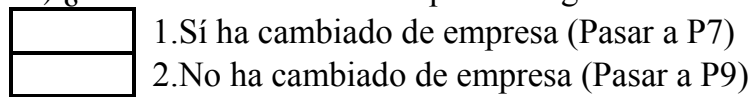

P7. Hacer solo si P6=1. Dentro de su nueva empresa, ¿ocupa usted el mismo puesto de trabajo y/o las mismas funciones que en la anterior?

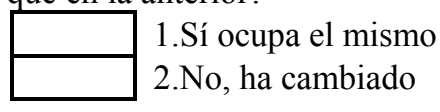

P8. Hacer solo si P1=1. Dentro de su nueva empresa, ¿ha recibido una mejora salarial en comparación con la empresa anterior?

1.Sí (Pasar a P11)

2.No (Pasar a P11)

P9. Hacer solo si P1=1. Desde la fecha X (encuestador tener en cuenta la fecha de finalización de la última acción formativa) ¿le han ascendido, ocupa usted un puesto más alto jerárquicamente?

1-Sí P9.1. ¿Cuál?

\begin{tabular}{|l|l|}
\hline & 1.Directivo \\
\hline & 2.Mando intermedio \\
3.Técnico \\
4.Puesto cualificado \\
5.Puesto no cualificado \\
\cline { 1 - 1 } 98.Otro ¿Cuál? \\
99.Ns/Nc
\end{tabular}

2-No

P10. Hacer solo si P1=1. Desde la fecha X (encuestador tener en cuenta la fecha de finalización de la última acción formativa) ¿ha cambiado usted de funciones o responsabilidades?

1-Sí P10.1. ¿En qué se traduce ese cambio?

\begin{tabular}{|l|l}
\hline & 1.Tengo más funciones o funciones distintas \\
2.Tengo más responsabilidades o son distintas \\
98.Otro ¿Cuál?
\end{tabular}

2-No

P11. Hacer solo si $P 1=1$. ¿Existe en su empresa un mecanismo/sistema para la evaluación del desempeño en su puesto de trabajo?

1-Sí P11.1. ¿En qué medida ha variado su desempeño desde la la fecha X (encuestador tener en cuenta la fecha de finalización de la última acción formativa)? No leer la opción 3.

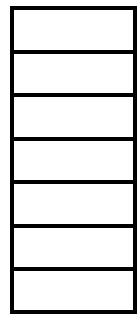

1.Ha empeorado mucho

2.Ha empeorado bastante

3.No ha cambiado, sigue igual

4.Ha mejorado bastante

5. Ha mejorado mucho

98.Otro ¿Cuál?

99. Ns/Nc

2-No/NsNc P11.2. ¿En qué medida ha variado su desempeño desde la fecha X (encuestador tener en cuenta la fecha de finalización de la última acción formativa)? No leer la opción 3.

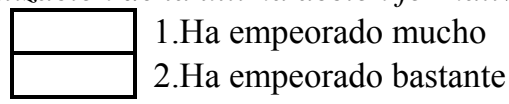




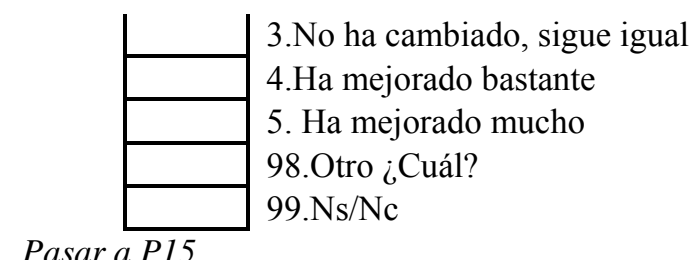

P12. Hacer sólo si $P 1=2$ ¿Desde cuándo lleva usted en esta situación laboral, desde cuándo está usted desempleado? Especifique cuánto tiempo

P13. Hacer solo si $P 1=2$. Aunque actualmente esté usted desempleado, ¿había trabajado anteriormente?

1-Sí P13.1. ¿Cuál era la actividad de la empresa en la que estaba usted trabajando? ¿Cuál era su sector productivo?

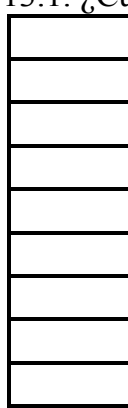
1.Ahorro
2.Artes gráficas
3.Banca
5.Limpieza
6.Seguridad
98.Otro: ¿Cual?
99.Ns/Nc

4.Medios de comunicación/actividades culturales o deportivas

7.Seguros, Oficinas y Despachos

\section{$2-\mathrm{No} / \mathrm{Ns} / \mathrm{Nc}$}

P14. Hacer solo si $P 1=2$. En una escala del 1 al 5 , donde 1 es igual a nada y 5 mucho, ¿en qué medida cree que la formación recibida ha facilitado o facilitará su incorporación al mercado laboral? Encuestador, no leer la opción 3.

\begin{tabular}{|l|l}
\hline & 1.Nada \\
& 2.Poco \\
& 3. Ni mucho ni poco \\
& 4.Algo \\
\hline & 5. Bastante
\end{tabular}

P15. Hacer a todos. Sugerencias. ¿Desea realizar cualquier sugerencia u observación?

AGRADECIMIENTO Y DESPEDIDA

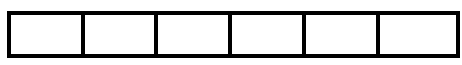

Fecha de realización

\section{ABOUT THE AUTHORS / SOBRE LOS AUTORES}

Ramírez-del-Río, Antonio (antonio.ramirez@systeme.es). Licenciado en Psicología y Especialista en Valoración de programas por la Universidad Autónoma de Madrid. Master en Dirección de Recursos Humanos. Es el autor de contacto para este artículo. Sus principales líneas de trabajo son la evaluación del impacto y de la calidad de la formación. Trabaja en Systeme, Innovación y Consultoría. Su dirección postal es: C/ Arce, 18, 28210-Valdemorillo (Madrid).

Garrido Casas, Jorgina (jorgina.garrido@systeme.es). Doctora en Psicología, especializada en consultoría de Recursos Humanos. Sus principales líneas de trabajo son la evaluación del impacto y de la calidad de la formación. Trabaja en Systeme, Innovación y Consultoría. Su dirección postal es: C/ Arce, 18, 28210-Valdemorillo (Madrid). 
Ramírez-del-Río, Antonio \& Garrido Casas, Jorgina (2011). Evaluación del impacto del esfuerzo formativo en la empleabilidad de los trabajadores en el contexto del modelo formativo tripartito español. RELIEVE, v. 17, n. 2 , art. 4.

http://www.uv.es/RELIEVE/v17n2/RELIEVEv17n2_4.htm

\section{$\underline{\text { ARTICLE RECORD / FICHA DEL ARTÍCULO }}$}

\begin{tabular}{|c|c|}
\hline $\begin{array}{l}\text { Reference } \\
\text { /Referencia }\end{array}$ & $\begin{array}{l}\text { Ramírez-del-Río, Antonio \& Garrido Casas, Jorgina (2011). Evaluación del impacto del esfuerzo forma- } \\
\text { tivo en la empleabilidad de los trabajadores en el contexto del modelo formativo tripartito español. } \underline{R E-} \\
\text { LIEVE, v. } 17, \text { n. } 2 \text {, art. } 4 \text {. http://www.uv.es/RELIEVE/v17n2/RELIEVEv17n2 } 4 . h t m\end{array}$ \\
\hline Title / Título & $\begin{array}{l}\text { Evaluación del impacto del esfuerzo formativo en la empleabilidad de los trabajadores en el contexto del } \\
\text { modelo formativo tripartito español. [Assessment of training effects on employability within the institu- } \\
\text { tional Spanish training model]. }\end{array}$ \\
\hline \begin{tabular}{l|l} 
Authors / \\
Autores
\end{tabular} & Ramírez-del-Río, Antonio \& Garrido Casas, Jorgina \\
\hline \multicolumn{2}{|c|}{ Review / Revista RELIEVE (Revista ELectrónica de Investigación y EValuación Educativa), v. 17, n. 2} \\
\hline ISSN & $1134-4032$ \\
\hline $\begin{array}{l}\text { Publication date } \\
\text { / Fecha de } \\
\text { publicación }\end{array}$ & $\begin{array}{l}2011 \text { (Reception Date: } 2011 \text { March 14; Approval Date: } 2011 \text { December 20. Publication Date: } 2011 \\
\text { December 20). }\end{array}$ \\
\hline $\begin{array}{l}\text { Abstract / } \\
\text { Resumen }\end{array}$ & $\begin{array}{l}\text { The association between quantity of training and employability was analysed through a non experimental } \\
\text { research with two non equivalent groups. The operationalization of training quantity is considered to } \\
\text { provide an indicator of training effort. Thus, training effort (number of courses) was analysed as the in- } \\
\text { dependent variable and employability as dependent variable (occupation indicators, activity enhance- } \\
\text { ment and job performance). The results show that training is related to activity enhancement, specially } \\
\text { when workers belong to a small/medium enterprise (SME) and/or to technical or qualified positions. A } \\
\text { positive association between training and performance also appeared in the case of SMEs. } \\
\text { Se analiza la asociación entre cantidad de formación y empleabilidad mediante una investigación no ex- } \\
\text { perimental con dos grupos no equivalentes. Se considera que la operativización de la cantidad de forma- } \\
\text { ción proporciona un indicador próximo al esfuerzo formativo realizado. Así, se estudió la variable inde- } \\
\text { pendiente esfuerzo formativo (número de cursos) y la dependiente, empleabilidad (indicadores de ocupa- } \\
\text { ción, enriquecimiento de actividad y desempeño). Los resultados muestran que la formación está asociada } \\
\text { al enriquecimiento de la actividad, sobre todo si los trabajadores pertenecen a una PYME y/o a puestos } \\
\text { técnicos o cualificados. También hay una asociación positiva entre formación y desempeño en PYMES. }\end{array}$ \\
\hline $\begin{array}{l}\text { Keywords / } \\
\text { Descriptores }\end{array}$ & $\begin{array}{l}\text { Employability, labour market, social outcome, profesional training, training paradigm, training assess- } \\
\text { ment, training results, training impact. } \\
\text { Empleabilidad, mercado de trabajo, retorno social, formación profesional, paradigma de formación, eva- } \\
\text { luación de la formación, resultados de la formación, impacto de la formación }\end{array}$ \\
\hline $\begin{array}{l}\text { Institution / } \\
\text { Institución }\end{array}$ & Systeme, Innovación y Consultoría. Madrid (España). \\
\hline $\begin{array}{l}\text { Publication site / } \\
\text { Dirección }\end{array}$ & http://www.uv.es/RELIEVE \\
\hline $\begin{array}{l}\text { Language / } \\
\text { Idioma }\end{array}$ & Español \& English version (Title, abstract and keywords in English \& Spanish) \\
\hline
\end{tabular}

\section{RELIEVE}

\section{Revista ELectrónica de Investigación y EValuación Educativa E-Journal of Educational Research, Assessment and Evaluation}

[ISSN: 1134-4032]

(C) Copyright, RELIEVE. Reproduction and distribution of this articles it is authorized if the content is no modified and their origin is indicated (RELIEVE Journal, volume, number and electronic address of the document).

(C) Copyright, RELIEVE. Se autoriza la reproducción y distribución de este artículo siempre que no se modifique el contenido y se indique su origen (RELIEVE, volumen, número y dirección electrónica del documento). 